\title{
Internet of Things for Healthcare Using Effects of Mobile Computing: A Systematic Literature Review
}

\author{
Shah Nazir $\mathbb{D}^{1},{ }^{1}$ Yasir Ali, ${ }^{1}$ Naeem Ullah, ${ }^{1}$ and Iván García-Magariño $\mathbb{D}^{2}$ \\ ${ }^{1}$ Department of Computer Science, University of Swabi, Ambar, Pakistan \\ ${ }^{2}$ Department of Software Engineering and Artificial Intelligence, Complutense University of Madrid, Madrid, Spain
}

Correspondence should be addressed to Shah Nazir; snshahnzr@gmail.com

Received 22 August 2019; Accepted 22 October 2019; Published 14 November 2019

Academic Editor: Miguel Garcia-Pineda

Copyright (c) 2019 Shah Nazir et al. This is an open access article distributed under the Creative Commons Attribution License, which permits unrestricted use, distribution, and reproduction in any medium, provided the original work is properly cited.

The impact of Internet of Things has been revolutionized in all fields of life, but its impact on the healthcare system has been significant due to its cutting edge transition. The role of Internet of Things becomes more dominant when it is supported by the features of mobile computing. The mobile computing extends the functionality of IoT in healthcare environment by bringing a massive support in the form of mobile health ( $\mathrm{m}$-health). In this research, a systematic literature review protocol is proposed to study how mobile computing assists IoT applications in healthcare, contributes to the current and future research work of IoT in the healthcare system, brings privacy and security in health IoT devices, and affects the IoT in the healthcare system. Furthermore, the intentions of the paper are to study the impacts of mobile computing on IoT in healthcare environment or smart hospitals in light of our systematic literature review protocol. The proposed study reports the papers that were included based on filtering process by title, abstract, and contents, and a total of 116 primary studies were included to support the proposed research. These papers were then analysed for research questions defined for the proposed study.

\section{Introduction}

The sudden rise in the population has brought many challenges in health services and ultimately it has led to the scarcity of medical resources. It is in dire need to address these challenges and provide a prompt solution based on the limited resources. The IoT, mobile, and network connectivity provide the best solution due to their less cost and easy-to-use features [1]. The main focus of Internet of Things- (IoT-) based healthcare services is to provide a rich user experience at low cost and improve the quality of life [2]. The major function of IoT is to provide connectivity to the available medical resources and reliable, effective, and smart healthcare services to the aged patients who suffer from chronic diseases. The IoT brings smart healthcare system in the medical field which is usually composed of sensors with smart functionality, a remote server, and the network. This system is focused to provide monitoring with multi-dimensional features and basic treatment suggestion [3].
Similarly, the mobile computing provides services to IoT by using mobile phone services, apps, or through $\mathrm{m}$-health care system. The $\mathrm{m}$-health contributes to the IoT by furnishing various services like compactness, IP connectivity, low-power consumption, and security [4]. In recent time, numerous applications have been developed that are intended to deliver mobile-based services to the users in the field of healthcare. The smartphone applications enable the patients to know about their diseases after the analysis in the fields of gynaecology and paediatrics [5]. The application of IoT in healthcare is categorized as single condition applications and clustered condition applications. A few applications discussed in this paper are glucose level sensing, blood pressure monitoring system, body temperature monitoring, oxygen saturation monitoring system, ECG (electrocardiogram) monitoring system, wheelchair management system, and rehabilitation system. Some papers are available on IoT in healthcare but these are concerned with only IoT in healthcare. However, the proposed research is concerned with IoT in healthcare using the concept of 
mobile computing, and the proposed research is a comprehensive analysis of the existing research.

The contribution of the proposed study is to offer a systematic literature review protocol to study how mobile computing assists IoT applications in healthcare, contributes to the current and future research work of IoT in the healthcare system, brings privacy and security in health IoT devices, and affects the IoT in the healthcare system. The proposed study reports the papers that were included based on filtering process and contents, and a total of 116 primary studies were included to support the proposed research. These papers were then analysed for research questions defined for the proposed study.

This research paper is organized in such a manner that research methodology is described next to the first part of the paper which is introduction. In research methodology section, research questions and their description, research strategy, and inclusion and exclusion criteria for research papers are defined in detail. A list of the selected research papers is also shared in this section. Qualitative and quantitative analysis along with the appropriate figure is also a part of this section. An overview of the selected studies and detail of the research questions are organized in the third section of this paper. Certain types of limitations defined based on the research study are the fourth part of this paper. At the end of this paper, some suggestions for the betterment of the IoT are proposed.

\section{Research Methodology}

In this paper, a systematic literature review (SLR) protocol is followed for reviewing papers from the most reliable sources. The SLR is mainly focused to find, interpret, and evaluate those research results that were addressing the formulated research questions. For the purpose of obtaining the research results from primary studies, both automatic and manual searches were done. Mainly the impacts/support, research work, applications, and security features provided by IoT in the healthcare system based on the support of mobile computing technology were considered. The primary studies went through quality assessment for the purpose of analysis of the study to capture the best fit results. Both forward and backward snowball procedures were applied in a bid to get the most pertaining results to our topic. The research followed the SLR in order to address the questions because a predefined protocol is necessary to reduce the possibility of researcher bias [6]. The prime focus of conducting an SLR is a comprehensive review of the included studies in a specific area to identify the gaps in existing research with the intention to further investigate and to furnish the depth understanding of the new phenomenon [7]. During the SLR process, various software tools like excel and SPSS were used for the purpose of analysis. The SLR protocol used in this study was based upon [8]. The steps that were followed during our SLR execution are organized according to Figure 1 , in which the first step is define the review protocol, the second is to define the search strategy, and the third is the documentation of the search strategies. Inclusion and exclusion criteria of the research papers are described in the fourth section of this paper, and quality criteria assessment is defined in the fifth section, while the quantitative metaanalysis is the last section of this paper.

The following sections briefly show the details of the protocol.

2.1. Defining Review Protocol. The SLR was intended to understand and review the experimental proof regarding the proposed research. The focus of the current research work was to formulate the research questions that provided the starting point for further investigation. The trends of IoT in the medical field have drastically changed the working of medical procedures. The application of IoT in healthcare has been an interesting area of study, so the SLR is mainly focused to formulate such questions which could answer that how IoT has affected the healthcare system with the support of mobile computing. The following research questions given in Table 1 indicate that how IoT with the support of using mobile computing contributes towards the healthcare system in smart hospitals or the healthcare system.

2.2. Defining Search Strategy. We performed both automatic and manual searches for the purpose of fetching the most relevant results. Our search strategy started from an automatic search that was performed in the electronic databases and confirmed by experts in the field of IoT, medical, and mobile computing. These databases are listed in Table 2. The reason for selection of these libraries was that they include high-quality networking journals and proceedings of conferences. Our search started from the period of 2011 and it was finished in 2019.

Search term or keywords were defined based on the following steps [9]:

(a) The major terms were derived from research questions

(b) Alternating spellings or synonyms were identified for the major terms

(c) Keyword was identified from books and relevant research papers

(d) Boolean operator OR was used for synonyms or alternating spellings

(e) Major terms were linked by Boolean AND operator

The search string was formed after the analysis of the following keywords in order to retrieve the relevant information from the mentioned databases. Keywords are "Internet of Things," "Healthcare," "Smart hospitals," and "Mobile computing." The search string was formed based on combining the keywords in the following ways:

(Internet of Things OR IoT) AND (Smart hospitals) AND (Healthcare) AND (Mobile Computing)

OR

"Internet of Things OR IoT" and "Smart hospitals" and "healthcare" and Mobile computing" 


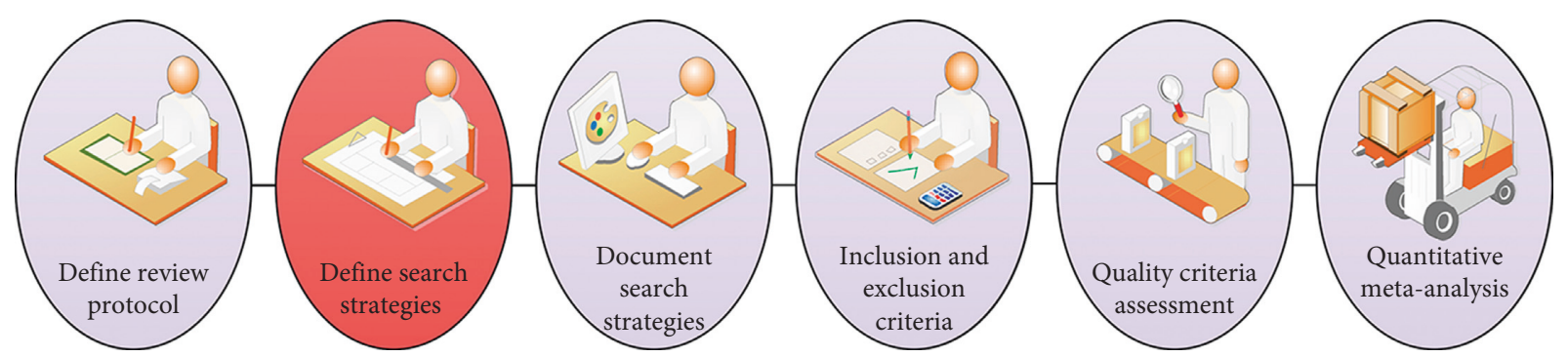

FIgURE 1: SLR protocol design.

TABLE 1: Research questions, their description, and motivation.

Research question

RQ-1. How mobile computing affects the Internet of Things in healthcare?

RQ-2. How the mobile computing secures the IoT devices in the healthcare system?

RQ-3. What are applications of IoT in the healthcare system by using mobile computing?

RQ-4. What are available research works in IoT for healthcare using mobile computing?
Description and motivation

The main focus of this question is to find out that how mobile computing provides a basic platform and services for the IoT in the healthcare system or smart hospitals

The question is aimed to investigate into those security and privacy measures and features that are provided by mobile computing to IoT devices in the healthcare system

The main idea is to investigate into depth detail about IoT applications in the healthcare system by the support of mobile computing, and how mobile computing provides a user-friendly interface experience to IoT devices in healthcare environment This question is formulated to study the existing work by IoT accompanied by mobile computing in the medical health system
TABLE 2: Libraries searched.

\begin{tabular}{l}
\hline ACM \\
\hline ScienceDirect \\
Springer \\
IEEE \\
Taylor and Francis \\
Hindawi \\
\hline
\end{tabular}

The aforementioned search string was checked on each database and its pattern was modified based on retrieving the best relevant results. A trial search was made to check the validity of the effectiveness of the search strategy.

2.3. Document Search Strategy. In this step of SLR design, a proper documentation of all the search strategies was recorded. The output of this step was a document which was containing all of the information about search strategy. The contents of the search strategy document were like date of search, name of online library used, search strategies, types of sources, number of results retrieved, filtered, name of keywords, and their combinations. All the records about search results were documented in the form of a report.

2.4. Criteria for Inclusion and Exclusion. The criteria for inclusion and exclusion to answer the questions for research papers are briefly listed in Table 3 .
The selection of papers was completed in three steps. These steps are as follows: in the first step, the duplication and redundant papers were removed; the next step was to read the keyword, abstracts, and titles of research papers, and the last step was full reading of research papers. At last, both steps, the inclusion and exclusion criteria were applied. To remove the biasness, the conflict analysis was performed at the end of step-3. The procedure of SLR, quality assessment, and selection of studies has been depicted in Figure 2. In the first step of SLR, duplicates or repeated papers were removed. In step-2, selection of paper was based on reading titles and abstracts. In step-3, full reading of paper was performed. The procedure of quality assessment came in step-4. In step-5, snowball procedure was performed. This complete process is depicted in Figure 2.

2.5. Snowballing. The snowball procedure used in the proposed study was based on Wohlin Guideline [9]. Both forward and backward snowballing were performed in order to capture the most relevant research papers by analyzing the reference of each paper. In snowballing, there are four steps involved. These steps are as follows: in step-1, initially 200 papers were identified, and then in step-2, after reading the titles, this figure becomes 110. After step-3, it reduced to 43, and in the final step it, becomes 12 papers; these papers went for the quality assessment and were added to 104. Finally, a list of 116 papers was identified for studies. 
TABLE 3: Inclusion and exclusion criteria.

\begin{tabular}{lc}
\hline Inclusion criteria & \multicolumn{1}{c}{ Exclusion criteria } \\
\hline All papers that published in English were selected \\
$\begin{array}{l}\text { Focus was to select primary studies like original } \\
\text { research papers }\end{array}$ & Papers other than English language \\
$\begin{array}{l}\text { Only relevant papers were selected that contained } \\
\text { empirical proofs }\end{array}$ & Papers that did not define the topic properly were excluded \\
Latest research papers, i.e., 2011 to 2019, were selected \\
for studies
\end{tabular}

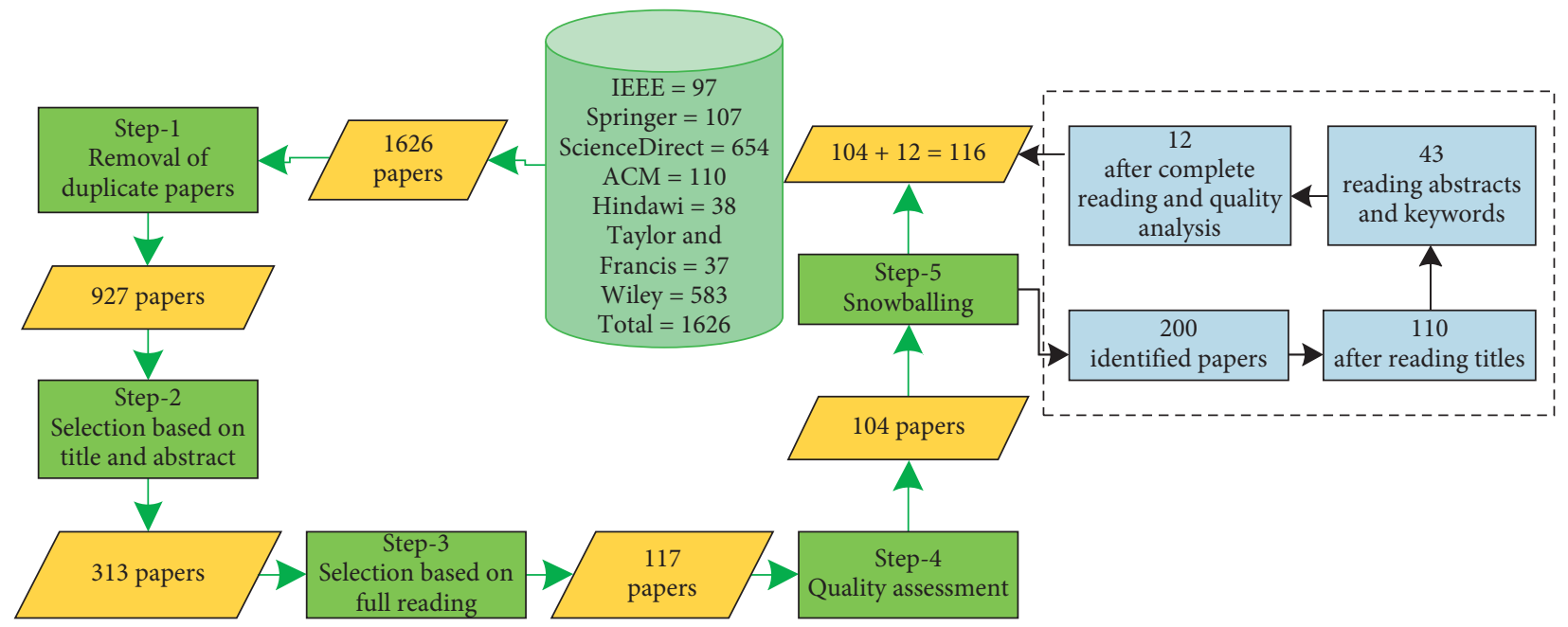

FIGURE 2: Flowchart of paper selection.

In our research, the following research papers were considered for the study after doing the search process. The list of research papers which was selected in the proposed research is listed in Table 4.

2.6. Quality Criteria Assessment. In quality assessment criteria, we analysed all the collected studies based upon the coherence and relevancy of addressing the research questions defined. For the said purpose, we analysed the contents of each paper, and based on thorough studies, we scored them by " 1 " and "0.5." These values were interpreted as " 1 " = highly relevant and " $0.5 "=$ medium level of detail. We scored each research paper based on these values against the research questions RQ-1, RQ-2, RQ-3, and RQ-4 and their total score, as depicted in Figure 3.

2.7. Quantitative Metadata Analysis. This is the last step of SLR protocol design, and it was conducted to perform some statistical analyses on quantitative data. It should be noted that the literature on quantitative meta-analysis also typically recommends establishing research quality criteria for inclusion decisions [121]. The details of results included and excluded from each library have been depicted in Figure 4.

In this step, we quantitatively analysed and summarised the findings from the multiple sources like journals, conferences and book sections. Then, we perform some statistical quantitative analyses on the data in order to know more about research questions and trends. The trends in IoT healthcare based on using the support of mobile computing have been displayed. The research work has been presented based on the number of papers per year, refers to Figure 5.

Similarly, the sources of research have been identified and presented in Figure 4. The distribution of sources was divided in such a way that the number of papers from journals as $61(62 \%)$, number of papers from conference proceedings as $34(33 \%)$, number of extractions from book chapters as $5(5 \%)$, and from workshops as $2(2 \%)$, as depicted in Figure 6.

The quantitative meta-analysis does not end here but also documented the details about the number of exclusion and inclusion of papers.

\section{Overview of Selected Studies and Results}

The following are the research questions that were answered in detail by keeping the objectives of questions in mind. Our research questions are RQ-1, RQ-2, RQ-3, and RQ-4. In RQ1 , the effects of mobile computing in IoT-based healthcare were explained in light of $\mathrm{m}$-health. In RQ-2, the privacy and security features provided by mobile computing in the IoT healthcare system were elaborated. In RQ-3, the various applications based on mobile computing in the IoT-based healthcare system were explained. In RQ-4, the trends in the field healthcare system by IoT with the support of mobile computing were explained in depth. The following are the detail answer of each research question. 
TABLE 4: List of selected papers.

\begin{tabular}{|c|c|c|}
\hline S. No & Ref\# & Title \\
\hline 1 & {$[10]$} & A healthcare monitoring system using random forest and internet of Things (IoT) \\
\hline 2 & {$[11]$} & Smart Healthcare: An Approach for Ubiquitous Healthcare Management Using IoT \\
\hline 3 & {$[12]$} & Smart ambulance system using concept of big data and internet of things \\
\hline 4 & {$[13]$} & Internet of things, smart sensors, and pervasive systems: Enabling connected and pervasive healthcare \\
\hline 5 & {$[14]$} & A hybrid framework for multimedia data processing in IoT-healthcare using blockchain technology \\
\hline 6 & {$[15]$} & $\begin{array}{c}\text { An approach to develop the smart health using Internet of Things and authentication based on biometric } \\
\text { technology }\end{array}$ \\
\hline 7 & {$[16]$} & $\begin{array}{c}\text { Context-aware anonymous authentication protocols in the internet of things dedicated to e-health } \\
\text { applications }\end{array}$ \\
\hline 8 & {$[17]$} & Edge computing for Internet of Things: A survey, e-healthcare case study and future direction \\
\hline 9 & {$[18]$} & Edge-of-things computing framework for cost-effective provisioning of healthcare data \\
\hline 10 & {$[19]$} & Enabling technologies for fog computing in healthcare IoT systems \\
\hline 11 & {$[20]$} & Harnessing the power of Internet of Things based connectivity to improve healthcare \\
\hline 12 & {$[21]$} & $\begin{array}{c}\text { Internet of Things (IoT), mobile cloud, cloudlet, mobile IoT, IoT cloud, fog, mobile edge, and edge emerging } \\
\text { computing paradigms: Disambiguation and research directions }\end{array}$ \\
\hline 13 & {$[22]$} & Internet of Things: A survey of enabling technologies in healthcare and its applications \\
\hline 14 & {$[5]$} & Mobile cloud computing based stroke healthcare system \\
\hline 15 & {$[23]$} & Mobile edge computing based QoS optimization in medical healthcare applications \\
\hline 16 & {$[24]$} & $\begin{array}{c}\text { Paradigm of IoT big data analytics in the healthcare industry: A review of scientific literature and mapping of } \\
\text { research trends }\end{array}$ \\
\hline 17 & {$[25]$} & Policy-based access control for constrained healthcare resources \\
\hline 18 & {$[26]$} & Smart integrated IoT healthcare system for cancer care \\
\hline 19 & {$[27]$} & Enabling mass surveillance: data aggregation in the age of big data and the Internet of Things \\
\hline 20 & {$[28]$} & $\begin{array}{c}\text { Applying a mindfulness-based reliability strategy to the Internet of Things in healthcare-A business model in } \\
\text { the Vietnamese market }\end{array}$ \\
\hline 21 & {$[29]$} & A Smart Healthcare Monitoring System Using Smartphone Interface \\
\hline 22 & {$[30]$} & A study on medical Internet of Things and Big Data in personalized healthcare system \\
\hline 23 & {$[31]$} & An IoT architecture for preventive maintenance of medical devices in healthcare organizations \\
\hline 24 & {$[32]$} & An IOT Based Smart Mobile Health Monitoring System \\
\hline 25 & {$[33]$} & Context-aware pseudonymization and authorization model for IoT-based smart hospitals \\
\hline 26 & {$[34]$} & EEG-based transceiver design with data decomposition for healthcare IoT applications \\
\hline 27 & {$[35]$} & Effectiveness of IoT in the Medical Field with Enhanced Features \\
\hline 28 & {$[36]$} & Exploring temporal analytics in fog-cloud architecture for smart office healthcare \\
\hline 29 & {$[37]$} & Fog assisted-IoT enabled patient health monitoring in smart homes \\
\hline 30 & {$[38]$} & $\begin{array}{c}\text { Contextual activity based Healthcare Internet of Things, Services, and People (HIoTSP): An architectural } \\
\text { framework for healthcare monitoring using wearable sensors }\end{array}$ \\
\hline 31 & [39] & Fog computing for Healthcare 4.0 environment: Opportunities and challenges \\
\hline 32 & {$[40]$} & $\begin{array}{c}\text { Designing an Internet-of-Things (IoT) and sensor-based in-home monitoring system for assisting diabetes } \\
\text { patients: iterative learning from two case studies }\end{array}$ \\
\hline 33 & {$[41]$} & Framework for E-Health Systems in IoT-based environments \\
\hline 34 & {$[42]$} & InLife: a platform enabling the exploitation of IoT and gamification in healthcare \\
\hline 35 & {$[43]$} & Intelligent and Energy Efficient Mobile Smartphone Gateway for Healthcare Smart Devices Based on 5G \\
\hline 36 & {$[44]$} & Intelligent healthcare systems assisted by data analytics and mobile computing \\
\hline 37 & {$[45]$} & IOT and AI in healthcare: A systematic literature review \\
\hline 38 & {$[46]$} & IoT based mobile healthcare system for human activity recognition \\
\hline 39 & {$[47]$} & IoT-based remote pain monitoring system: From device to cloud platform \\
\hline 40 & {$[48]$} & The application of internet of things in healthcare: a systematic literature review and classification \\
\hline 41 & {$[49]$} & Towards energy-aware fog-enabled cloud of things for healthcare \\
\hline 42 & {$[50]$} & $\begin{array}{c}\text { Trust based Security Solution for Internet of Things Healthcare Solution: An End-to-End Trustworthy } \\
\text { Architecture }\end{array}$ \\
\hline 43 & {$[51]$} & Ubiquitous Healthcare System Based on the Sensors Network and Android Internet of Things Gateway \\
\hline 44 & {$[52]$} & Healthcare IoT: Benefits, vulnerabilities and solutions \\
\hline 45 & {$[53]$} & Security and privacy analysis of mobile health applications \\
\hline 46 & {$[54]$} & Security and Privacy Issues with IoT in Healthcare \\
\hline 47 & [55] & FoG assisted secure De-duplicated data dissemination in smart healthcare IoT \\
\hline 48 & {$[56]$} & Security and privacy in the medical internet of Things: a review \\
\hline 49 & {$[57]$} & Device-to-device communication based IoT system: benefits and challenges \\
\hline 50 & {$[58]$} & From A to Z: Wearable technology explained \\
\hline 51 & [59] & Fog computing-based IoT for health monitoring system \\
\hline 52 & {$[60]$} & Intelligent community medical service based on Internet of Things \\
\hline
\end{tabular}


TABle 4: Continued.

\begin{tabular}{|c|c|c|}
\hline S. No & Ref\# & Title \\
\hline 53 & {$[61]$} & $\begin{array}{c}\text { Real-Time Cloud-Based Health Tracking and Monitoring System in Designed Boundary for Cardiology } \\
\text { Patients }\end{array}$ \\
\hline 54 & {$[62]$} & Internet of Health Things: Toward intelligent vital signs monitoring in hospital wards \\
\hline 55 & {$[63]$} & $\begin{array}{l}\text { A new computing environment for collective privacy protection from constrained healthcare devices to IoT } \\
\text { cloud services }\end{array}$ \\
\hline 56 & {$[64]$} & A Simulation Study of Response Times in Cloud Environment for IoT-Based Healthcare Workloads \\
\hline 57 & {$[65]$} & A systematic review on recent advances in m-health systems: deployment architecture for emergency response \\
\hline 58 & {$[66]$} & Agent Technology Based Modelization Systems for Healthcare \\
\hline 59 & {$[67]$} & Advanced internet of Things for personalised healthcare systems: A survey \\
\hline 60 & {$[68]$} & An IoT based robust healthcare model for continuous health monitoring \\
\hline 61 & {$[69]$} & Cloud-centric IoT based student healthcare monitoring framework \\
\hline 62 & {$[70]$} & Design and implementation of smart healthcare system using IoT \\
\hline 63 & {$[71]$} & Enabling technologies for the Internet of Health Things \\
\hline 64 & {$[72]$} & Enhancing healthcare using m-care box (monitoring non-compliance of medication) \\
\hline 65 & {$[73]$} & Enterprise architecture of mobile healthcare for large crowd events \\
\hline 66 & [74] & Health care monitoring system in Internet of Things (IoT) by using RFID \\
\hline 67 & {$[75]$} & Integrated IoT medical platform for remote healthcare and assisted living \\
\hline 68 & {$[76]$} & Internet of Thing based healthcare monitoring system \\
\hline 69 & [77] & IoT based low-cost distant patient ECG monitoring system \\
\hline 70 & {$[78]$} & IoT based smart health care system using CNT electrodes (for continuous ECG monitoring) \\
\hline 71 & [79] & IoT Interface for Healthcare applications \\
\hline 72 & {$[80]$} & Ethics of the health-related Internet of Things: a narrative review \\
\hline 73 & {$[81]$} & $\begin{array}{c}\text { On Design of a Fine-Grained Access Control Architecture for Securing IoT-Enabled Smart Healthcare } \\
\text { Systems }\end{array}$ \\
\hline 74 & {$[82]$} & An intelligent and secure health monitoring scheme using IoT sensor based on cloud computing \\
\hline 75 & {$[83]$} & $\begin{array}{c}\text { The impact of the hybrid platform of Internet of Things and cloud computing on healthcare systems: } \\
\text { Opportunities, challenges, and open problems }\end{array}$ \\
\hline 76 & {$[84]$} & Highly secure and efficient architectural model for IoT based health care systems \\
\hline 77 & {$[85]$} & Internet of Things: architectures, protocols, and applications \\
\hline 78 & {$[86]$} & Semantic interoperability in heterogeneous IoT infrastructure for healthcare \\
\hline 79 & [87] & Internet of Things meets mobile health systems in smart spaces: An overview \\
\hline 80 & {$[1]$} & A conceptual framework for IoT-based healthcare system using cloud computing \\
\hline 81 & {$[88]$} & $\begin{array}{c}\text { A fog based middleware for automated compliance with OECD privacy principles in internet of healthcare } \\
\text { things }\end{array}$ \\
\hline 82 & {$[89]$} & $\begin{array}{c}\text { A novel and secure IoT based cloud centric architecture to perform predictive analysis of users activities in } \\
\text { sustainable health centres }\end{array}$ \\
\hline 83 & {$[90]$} & A personal health index system with IoT devices \\
\hline 84 & [91] & A survey on IoT applications, security challenges and counter measures \\
\hline 85 & {$[92]$} & An IoT-cloud based wearable ECG monitoring system for smart healthcare \\
\hline 86 & [93] & Automated patient appointment reminder for cross-platform mobile application \\
\hline 87 & [94] & Effective ways to use Internet of Things in the field of medical and smart health care \\
\hline 88 & [95] & Keynote Talk: Harnessing Health IOT for Smart Healthcare \\
\hline 89 & {$[4]$} & Mobile health (m-health) system in the context of IoT \\
\hline 90 & [3] & The Internet of Things in healthcare: An overview \\
\hline 91 & [96] & Patient monitoring system based on Internet of Things \\
\hline 92 & [97] & $\begin{array}{c}\text { A comprehensive and scalable middleware for ambient-assisted living based on cloud computing and Internet } \\
\text { of Things }\end{array}$ \\
\hline 93 & [98] & IoT-based surveillance system for ubiquitous healthcare \\
\hline 94 & [99] & IoT healthcare analytics: The importance of anomaly detection \\
\hline 95 & {$[100]$} & Improving the validity of lifelogging physical activity measures in an Internet of Things environment \\
\hline 96 & {$[101]$} & Internet of nano-things healthcare applications: Requirements, opportunities, and challenges \\
\hline 97 & {$[102]$} & Internet of Things (IoT) driven U-healthcare system architecture \\
\hline 98 & {$[103]$} & SmartHealth-NDNoT: Named Data Network of Things for Healthcare Services \\
\hline 99 & {$[104]$} & Privacy and security in mobile health apps: a review and recommendations \\
\hline 100 & [2] & The Internet of Things for health care: a comprehensive survey \\
\hline 101 & {$[105]$} & An implementation of IoT for healthcare \\
\hline 102 & {$[106]$} & $\begin{array}{c}\text { Internet of Things (IOT) enabled smart autonomous hospital management system-A real world health care } \\
\text { use case with the technology drivers }\end{array}$ \\
\hline 103 & [107] & Internet of Things: Remote patient monitoring using web services and cloud computing \\
\hline 104 & {$[108]$} & Opportunities and challenges of the Internet of Things for healthcare: Systems engineering perspective \\
\hline
\end{tabular}


TABLE 4: Continued.

\begin{tabular}{|c|c|c|}
\hline S. No & Ref\# & Title \\
\hline 105 & [109] & Wearable Internet of Things: Concept, architectural components and promises for person-centered healthcare \\
\hline 106 & [110] & Ecosystem-driven design of in-home terminals based on open platform for the Internet-of-Things \\
\hline 107 & [111] & Ubiquitous data accessing method in IoT-based information system for emergency medical services \\
\hline 108 & [112] & $\begin{array}{c}\text { Drug identification and interaction checker based on IoT to minimize adverse drug reactions and improve } \\
\text { drug compliance }\end{array}$ \\
\hline 109 & [113] & $\begin{array}{c}\text { Ecosystem analysis in the design of open platform-based in-home healthcare terminals towards the Internet- } \\
\text { of-Things }\end{array}$ \\
\hline 110 & [114] & Security and privacy mechanism for health Internet of Things \\
\hline 111 & [115] & Application of ZigBee and RFID Technologies in Healthcare in Conjunction with the Internet of Things \\
\hline 112 & [116] & A survey on ambient-assisted living tools for older adults \\
\hline 113 & [117] & Bringing IoT and cloud computing towards pervasive healthcare \\
\hline 114 & [118] & Privacy and security issues in IoT healthcare applications for the disabled users a survey \\
\hline 115 & [119] & A proposal for using the Internet of Things concept to increase children's health awareness \\
\hline 116 & [120] & The potential of Internet of $m$-health Things " $m$-IoT" for non-invasive glucose level sensing \\
\hline
\end{tabular}

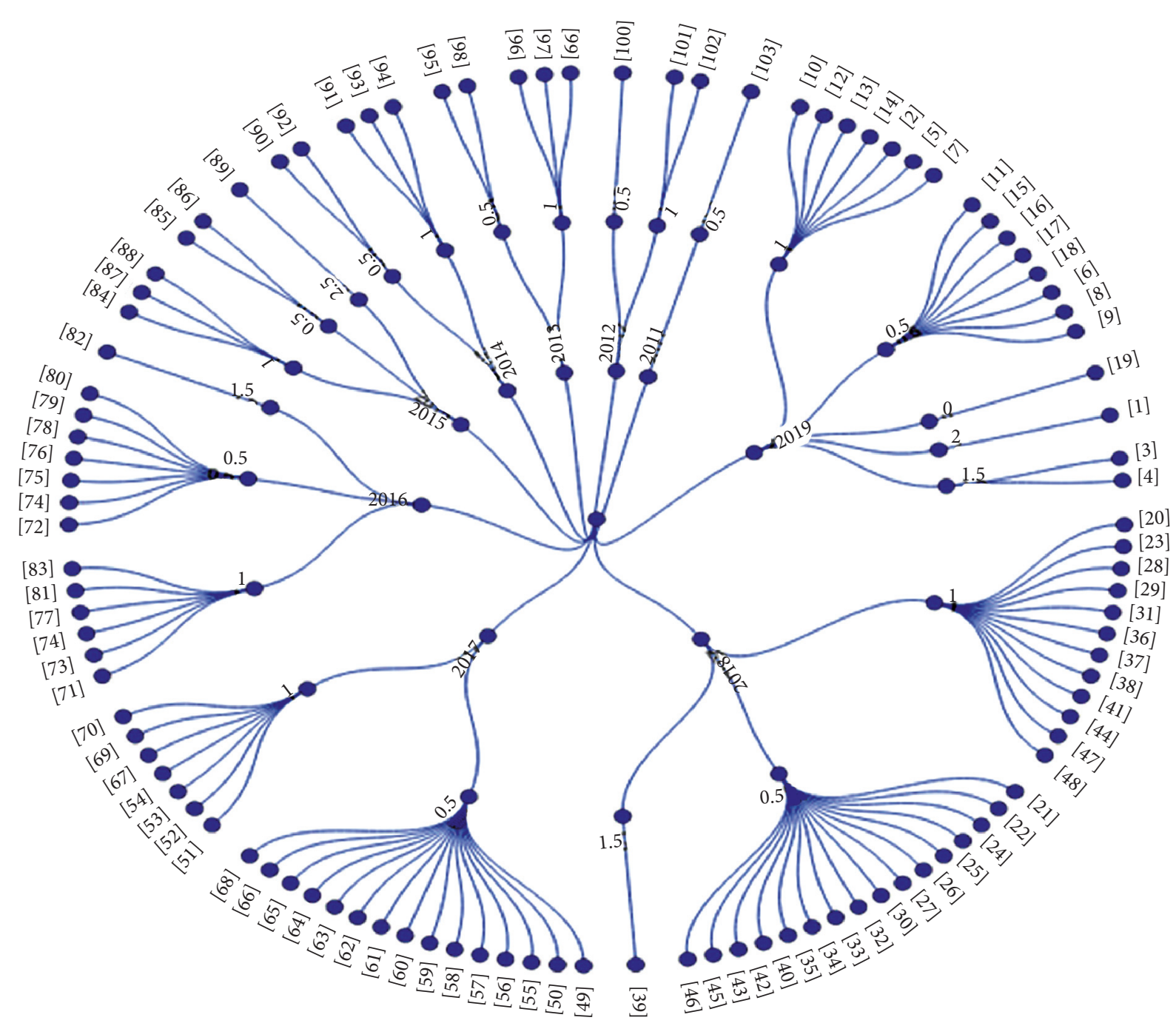

FIGURE 3: Quality assessment result.

3.1. RQ-1. How Mobile Computing Affects the IoT in Healthcare? In order to address this question, total 31 research papers were selected, and their composition of sources has been given in Figure 7. From 31 papers, 16 (52\%) papers were from conferences, 13 (42\%) were from journals, and $2(6 \%)$ were from book sections, as shown Figure 7.

The quality assessment result of RQ-1 has been depicted in Figure 7. In this figure, the " 1 value" represents the 


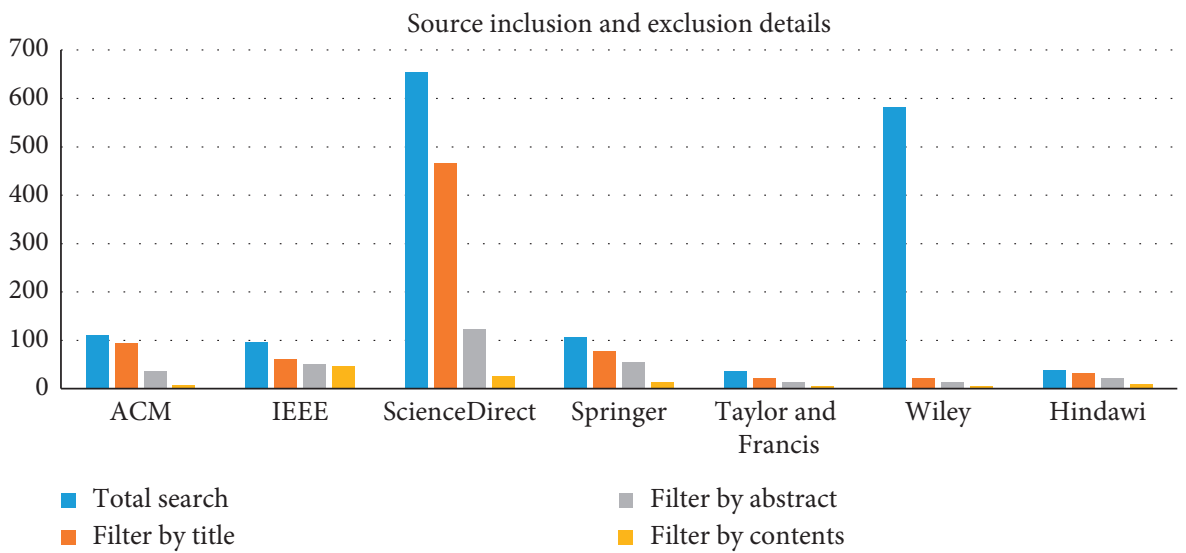

FIgURE 4: Source distribution.

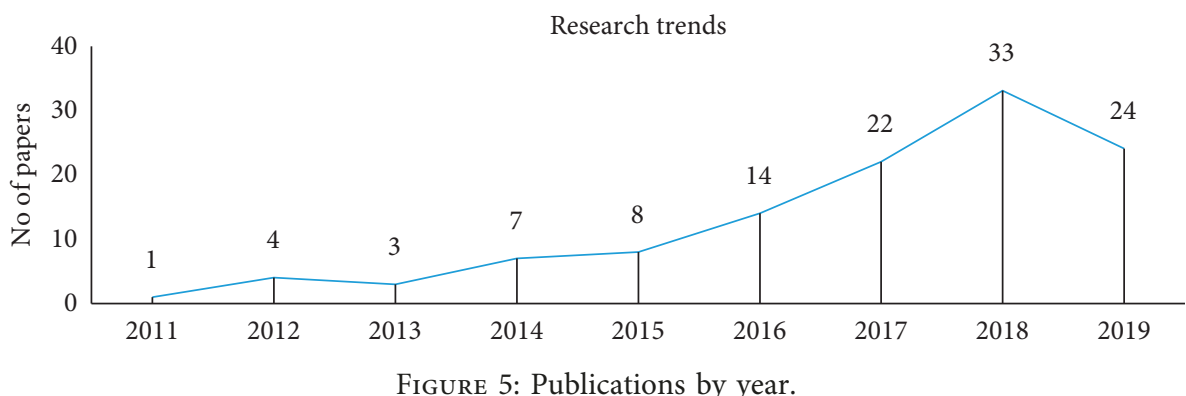

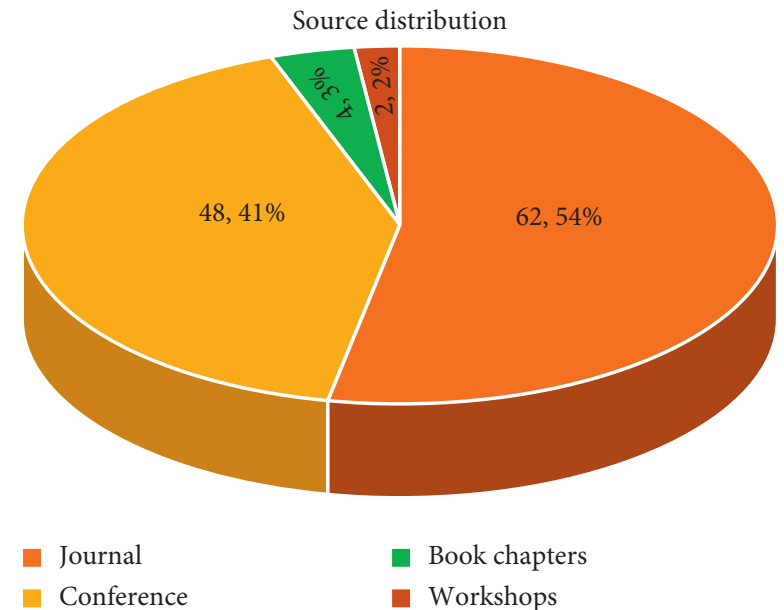

Figure 6: Source distribution.

maximum level of relevance pertaining to the providing details about answering RQ- 1 . The " 0.5 score" represents the moderate level of details in terms of details relevant to the answer of RQ-1, and "0 score" shows no details exist in the selected research papers. Among the selected papers for RQ1, 17 papers contain more detail, and 14 papers contain medium level of details. So, total 31 papers have been studied for the purpose of providing a comprehensive answer to RQ1 . The details provided by research papers in order to answer RQ-1 have been displayed in Figure 8.

Mobile computing is a new technological trend and its applications are in multiple disciplines and are involved in

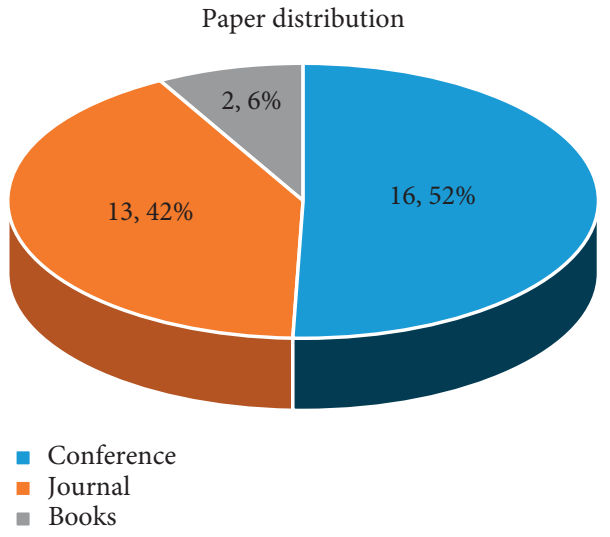

FIGURE 7: Selection of sources for RQ-1.

many areas. Mobile computing is about providing quality information services like processing, storage, information, and query to the phone users as well as users of laptops, pagers, and mobile phones that are geographically distantly located. Mobile computing is a type of new technology that enables computers and other information devices to send data without any physical connection to a fixed device. Internet of Things is an intelligent sensing technology that provides vital support for sending and receiving of mobile medical support [44]. The mobile computing affects the IoT by providing various services, applications, and devices. There are various mobile applications that have been developed by the research community for various areas of application. Two noticeable examples are $\mathrm{m}$-health and 


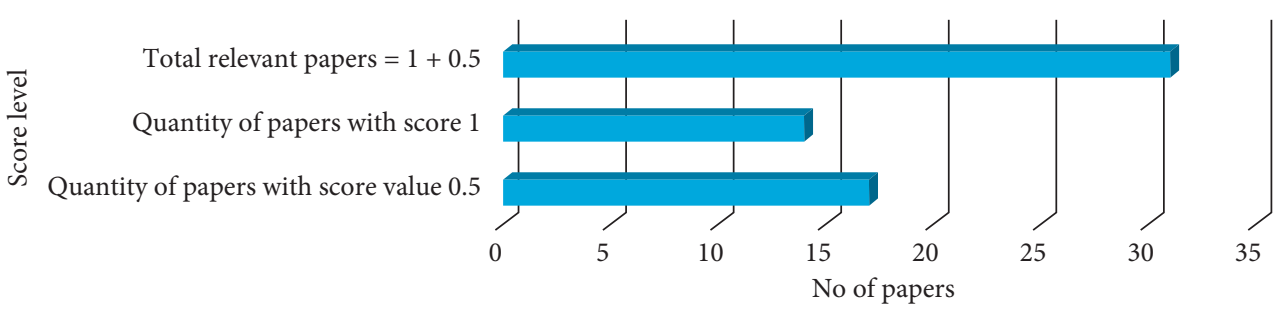

FIgUre 8: Quality assessment of RQ-1.

mobile learning (m-learning). Most $\mathrm{m}$-health applications are used by the healthcare professionals for various tasks like diagnosis of disease, drug reference, and medical calculations [21].

The concept of $\mathrm{m}$-health in the context of Internet of Things, combined form m-IoT, is applied in the health management system, and it produces the good results by making full and timely use of available and limited resources in order to provide best and more services to the patients. IoT-based $\mathrm{m}$-health services provide convenience to the patients by saving time and money without physical visits to the hospitals, unless their conditions become severe. These smart medical devices are capable to monitor various health parameters like level of sugar in blood, blood pressure, and pulse rate. These $\mathrm{m}$-health devices, for the purpose of doing these tasks, are connected to the IoT server. The list of those devices and their functionalities are given in Table 5 [4].

Mobile health is a medical health infrastructure that is formed by mobile devices and IoT, and it includes the uses of a mobile phone's core utility of general packet radio service (GPRS), 4G systems, global positioning system (GPS), and Bluetooth technology. M-health makes the decision based on the available data, and these data are gathered by using sensors in environment of IoT. The M-health dataset contains vital sign recordings and the movement of the body when it gets involved in many physical activities. These sensors can be placed at various positions of the body like the left ankle, right wrist, and chest to track the movement of the subject [46].

In order to provide an answer to RQ-1, the focus was to select those papers out of 112 covering the mobile-health or $\mathrm{m}$-health in context of IoT. So, from our collated studies, we identified 16 papers and their definition level of $\mathrm{m}$-health in light of IoT in healthcare scenario. For this purpose, among 16 papers, 10 (62\%) papers provided answer to RQ-2, 4 (25\%) answered to RQ-2 partially, and 2 (13\%) did not define any concepts pertaining to RQ-2, as shown in Figure 9.

These papers were analysed based on trends in $\mathrm{m}$-health in such a way that 2 papers from 2019, 6 papers from 2018, 2 papers from 2017, 1 paper 2016, and 2 from 2015 were selected, as given in Figure 10.

The use of mobile apps in IoT environment has been remained significant. Mobile apps provide a user interface to the data collected by various sensors or wearable devices. The most significant use of the mobile app is in PHS-personalized healthcare system, where various sensors such as accelerometers, gyroscopes, altimeters, and other low-cost portable devices are capable of being connected in an IoT environment. The concept of IoT-based PHS has become more popular due to the elevated rise of commercial wearable devices and mobile applications. These healthcare systems use a set of interconnected devices to create an IoT network for performing healthcare activities, such as diagnosis, monitoring, and surgeries at distance [67].

The mobile computing contributes to IoT by using the concept of smart healthcare. The smart healthcare is connected with low-power wireless technologies to build IoT, which becomes SmartHealthIoT. The patients can connect to the SmartHealthIoT through their smartphones, and the patients can be monitored, ubiquitously, either in their comfortable home environment or even on the move, by using the vital sign sensors connected to their smartphones. SmartHealthIoT is beneficial whenever the people require continuous monitoring and care, such as people with disabilities, aged, frail people living alone, children of dissimilar ages, and adults who are susceptible to near-fatal falls or sudden increases in blood pressure, heart rates, stress level, and so on. In case of emergency, a patient's location can be traced using his/her GPS coordinates. The SmartHealthIoT offers enhanced care and services at very low cost [103].

The modern mobile apps and third party API furnish limitless function in order to track the intensity of physical activity such as pace of walking. In this regard, the Fitbit is used which classifies the intensity of daily activities into categories like Very Active, Moderately Active, Lightly Active, and Sedentary; Moves records a series of walking segments containing duration, distance, and speed [100].

\subsection{RQ-2: How the Mobile Computing Secures the IoT Devices} in the Healthcare System? In order to answer RQ-2, the following research papers were found the top relevant based on abstract, keyword, and full reading. The detail of research papers that addressed RQ-2 is given in Table 6.

Score $1=$ represents concrete detail and $0.5=$ represents abstract details. In Table 7, the last column can be interpreted as High $=$ more relevant details, Medium = partial detail, and Low $=$ no detail or irrelevant. The distribution of relevancy level in the above table is like (relevancy level=high, 5), (relevancy level = medium, 4), and (relevancy level = low, 4), as depicted in Figure 10. Similarly, the paper relevancy to address RQ-1 has been depicted based on Table 7 in Figure 11.

In the healthcare system, there are many IoT devices such as sensors, heterogeneous wearable devices, gyroscopes, altimeters, or other wearable devices which transmit 
TABLE 5: m-health device description.

\begin{tabular}{|c|c|}
\hline m-health device name & Description and function \\
\hline TICKR & It is a heart rate monitoring device, which measures the heart rate by strapping across the chest \\
\hline FitBit surge & $\begin{array}{l}\text { Its function is to track the heart rate, pattern of sleeping pattern, and number of calories lost during } \\
\text { an exercise or in physical activity }\end{array}$ \\
\hline Forerunner 920XT & $\begin{array}{c}\text { It monitors the heart, a record of calorie calculation, step counter, swimming stroke counter, and } \\
\text { tracking elevation }\end{array}$ \\
\hline iBGSta & It measures the sugar and glucose level in blood, and its compatibility is good with iPhone and iPod \\
\hline
\end{tabular}

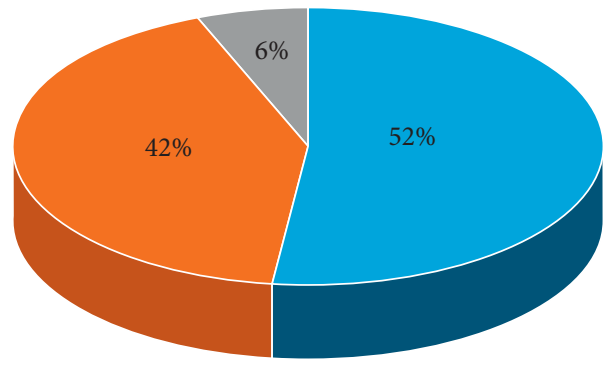

Definition present

Definition partially present

Definition not present

Figure 9: Level of detail.

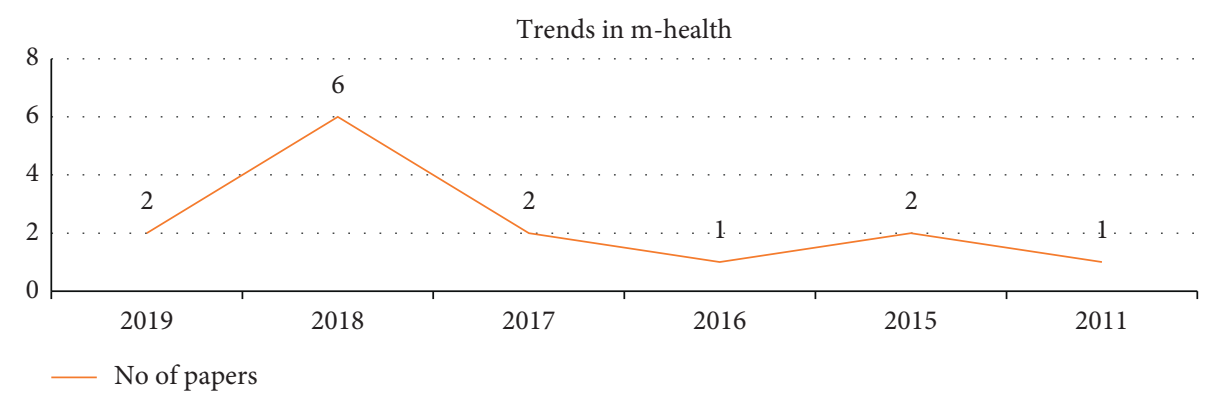

Figure 10: Paper distribution.

TABLe 6: RQ-2 details.

\begin{tabular}{llcc}
\hline Paper references & Year & Ref type & Relevancy level \\
\hline$[117,118]$ & 2012 & Journal & $1,0.5$ \\
{$[98]$} & 2016 & Journal & 1 \\
{$[53]$} & 2018 & Journal & 1 \\
{$[104]$} & 2015 & Journal & 1 \\
{$[56]$} & 2018 & Journal & 1 \\
{$[84]$} & 2017 & Conference & 1 \\
{$[52]$} & 2018 & Conference & 1 \\
{$[54]$} & 2018 & Journal & 1 \\
{$[50]$} & 2018 & Journal & 0.5 \\
{$[91]$} & 2016 & Conference & 0.5 \\
{$[4]$} & 2016 & Conference & 0.5 \\
\hline
\end{tabular}

a huge amount of data. So, we need a security mechanism to protect our data from unauthorised users. Similarly, if the patient's privacy is lost or compromised such as, especially, her identity of data, then it may have consequently lead to significant damages like physical, financial, and emotional harm to the patient. For this purpose, sophisticated m-health apps are able to sense changes in environmental and human body measurements in order to assess users' health and to generate alerts relevant to their condition [53]. In order to protect the privacy and security for patients in the healthcare environment based on IoT, a systematic mechanism is required. Hence, the mobile application solutions are 
TABLE 7: Healthcare applications using smartphone.

\begin{tabular}{|c|c|}
\hline Apps & Description \\
\hline Health Assistant & $\begin{array}{c}\text { Health Assistant keeps record of health parameters like weight, blood pressure, body temperature, } \\
\text { and other physical activities }\end{array}$ \\
\hline Healthy Children & Helps in finding a paediatrician in nearby location and requests for quick response \\
\hline Google Fit & Using sensors, it automatically tracks walking, running, and cycling activities \\
\hline Noom Walk & It is a health and fitness app like pedometer which counts user's steps \\
\hline Heart Rate Monitor & $\begin{array}{c}\text { This app allows a user to measure and monitor the heart rate at actual time and keep a record for } \\
\text { later analysis }\end{array}$ \\
\hline Eye $\mathrm{C}$ & This app helps in naturally improving vision by testing eye health information \\
\hline Blood Pressure Watch & This is a wearable device which collects, monitors, and keeps record of blood pressure data \\
\hline
\end{tabular}

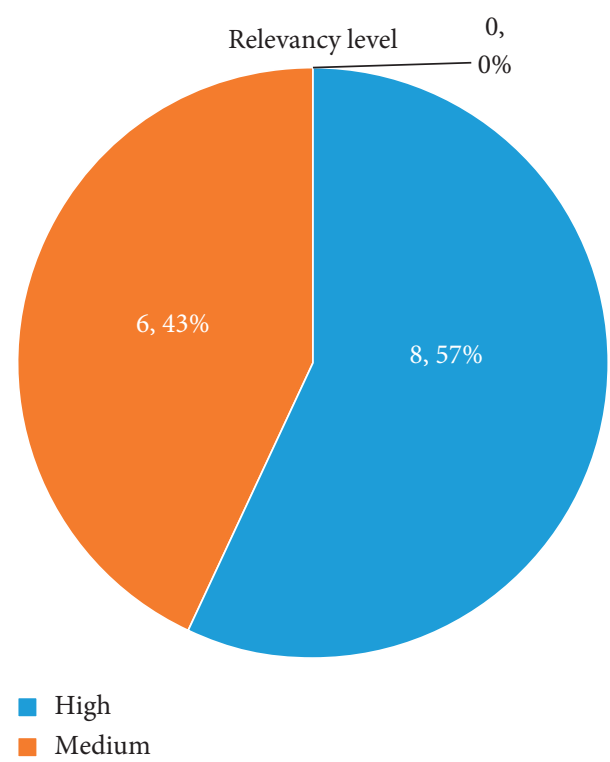

FIGURE 11: Composition of papers based on relevancy.

commonly considered due to their user-friendly features; so, secure healthcare service is a new demand for mobile solutions. A number of applications can be found in the healthcare sector with IoT technologies. The uprising of IoT is contributing towards modern healthcare from both economic and social perspectives in terms of prospects. Large user base and maturated ecosystem of traditional mobile internet service have significantly expedited towards the contribution of development of the IoT-powered inhome healthcare services, which is termed as Health-IoT. In various issues pertaining to health, numerous mobile applications are in use at Android market. Patient can also use the apps in order to measure, monitor, and record physical signs, such as blood pressure, glucose level in blood, and pulse rate. Smartphones are often used by the patient and medical personnel because they provide the facility of mobility and privacy protection [114]. The role of BodyGuardian Remote Monitoring System in IoT health-based environment is vital as IoT can be secured by using the BodyGuardian Remote Monitoring System. The system provides security in various fashions like first it separates patient identification information and observation data. Then, the system encrypts data on the device, during transmission and in storage. This technology is more helpful and can be used by two groups of people, i.e., first one is that needs an intensive level of medical need and second is the group of aged people who are suffering from chronic disease. The IoT devices send a huge amount of data to the servers and hence these devices are vulnerable to any sort of hacking attack like brute force attack and by using hard coding logins [54].

3.3. RQ-3: What Are the Applications of IoT in Healthcare Using Mobile Computing? In order to explain RQ-3, a total of 55 relevant papers were selected out of 55 research papers in which $22(40 \%)$ research assessment papers were from conference, 28 (51\%) research papers were from journal, 3 (5\%) were from books, and 2 (4\%) were from workshops, as depicted in Figure 12.

Similarly, the distribution of research papers in year-wise fashion for question RQ-3 has been displayed in Figure 13.

The level of detail found for RQ-3, from selected papers, has been presented in Figure 13. In 2011, abstract level of details has been found pertaining to our study. In 2012, the information extracted was not according to the metric. Similarly, in 2013 and 2014, the details found in research papers were partial to our study, and the level increased in 2015 and 2016. But, the level of details became more saturated in 2017, 2018, and 2019, as depicted in Figure 14.

There are various areas in which the intelligent applications have been developed. Although all these applications are not available, however, introductory research specifies that the potential of IoT is civilizing the lifestyle in our society. Some IoT applications are used in in-home automation systems, health monitoring systems, fitness tracking systems, environment protection, and smart cities [85]. Applications of IoT in healthcare have proved an enhancing well-being related to health and fitness. A lot of wearable devices are being developed to investigate a person's physical status. As the IoT is a prototype in which different objects intercommunicate through sensors, CPUs, and actuators to deliver a meaningful service [85]. Smart health system has been depicted in Figure 15.

Healthcare applications make it possible for patients and elder people to live independently. At this time, IoT sensors are being used to unceasingly check and record their health status and send warnings in case any irregular conditions were found. If some minor problems were detected, the IoT application itself may advise an adequate treatment to the patient. The following sections include various applications 


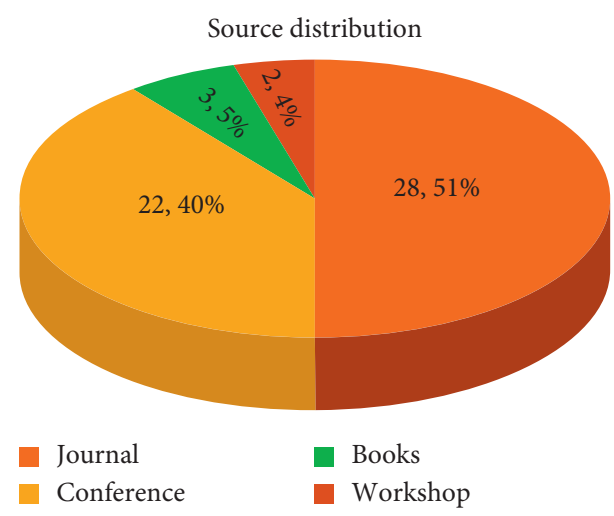

Figure 12: Selected research paper distribution and quality.

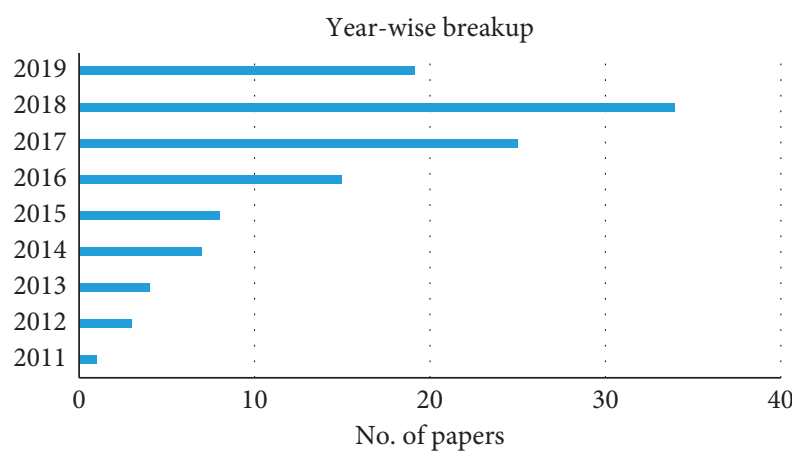

FIGURE 13: Year-wise breakup of research papers.

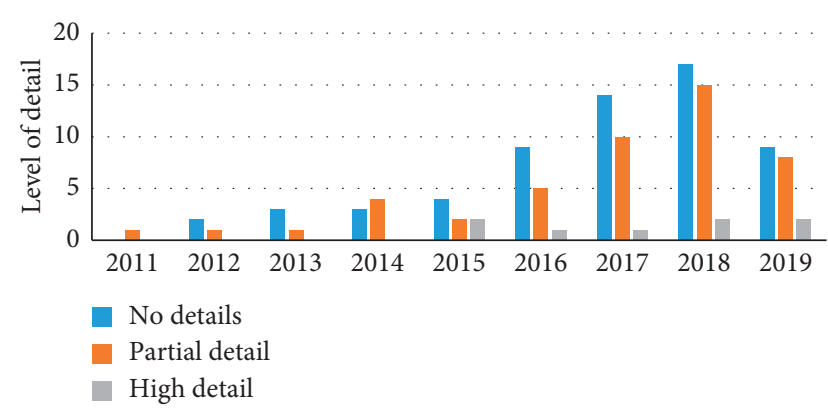

Figure 14: Year-wise breakup.

of IoT in healthcare services. IoT-based healthcare applications are divided into two broad categories: single condition applications and clustered condition applications, as shown in Figure 16 [2].

(i) Single condition applications: such applications are developed for a specific disease.

(ii) Clustered condition applications: the clustered condition applications can treat certain diseases together.

3.3.1. Single Condition Applications. Single condition applications are further categorised as follows:

(1) Glucose Level Sensing. Diabetes is a disease of metabolic in which the glucose level remains above the normal level for

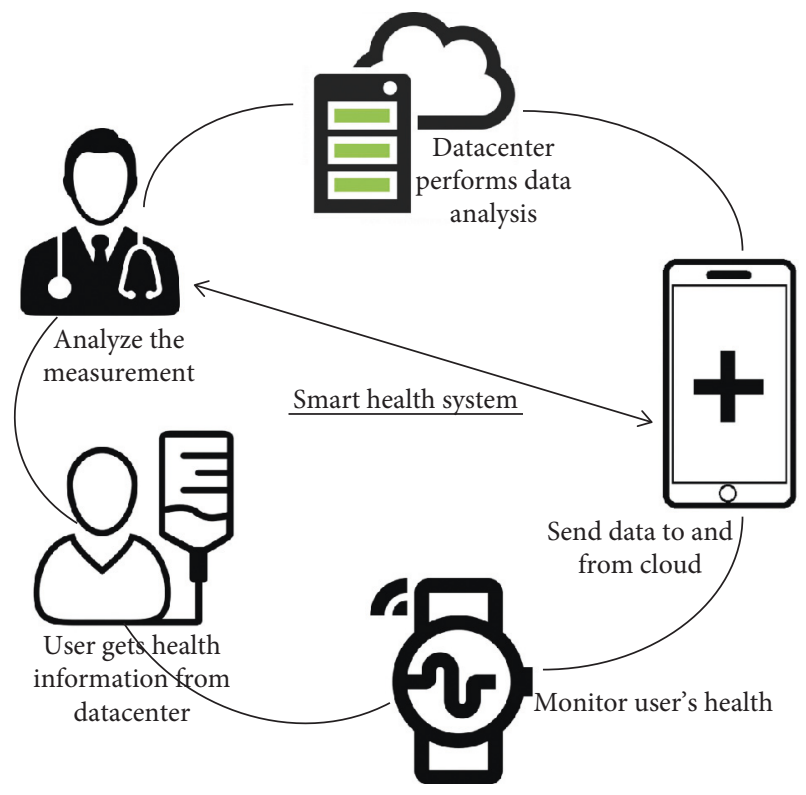

Figure 15: Applications of IoT in healthcare.

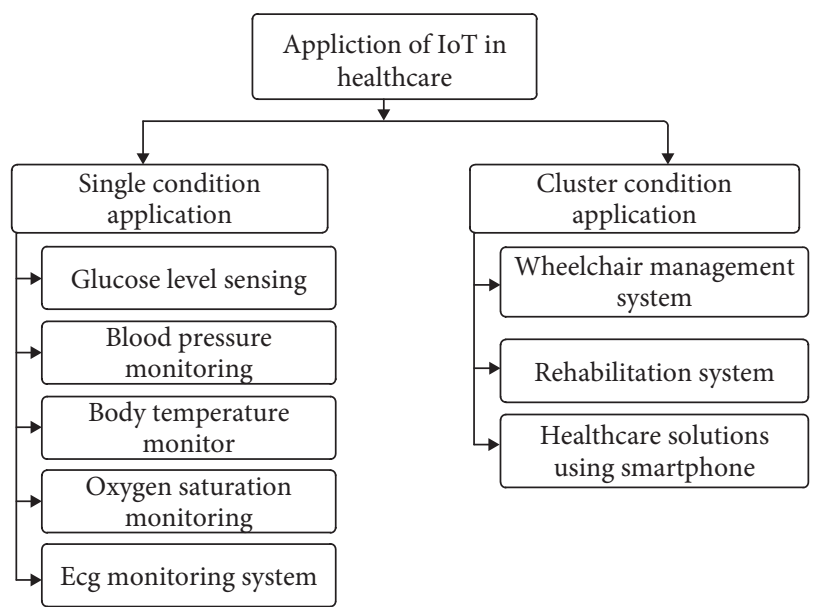

FIgURE 16: Classification of application in IoT-based healthcare.

a long period of time. Blood glucose monitoring system exposes an individual form of blood glucose and helps to suggest best planning of meals, required exercises, and medication times. An m-IoT configuration method for noninvasive glucose level measuring on actual basis is proposed.

According to this method, different sensors from patients are connected through IPv6 connectivity to the relevant healthcare providers. According to the utility model of [2], it discloses a transmission device based on IoT networks which transmits the collected corporal data on glucose level in blood. This device comprises a blood glucose collector, computer or smartphone, and processor. In addition, a general IoT-based medical acquiring detector for glucose level monitoring is proposed [2].

(2) Blood Pressure Monitoring System. Blood pressure specifies the force that the heart circulates blood around the 
body. A supportive IoT-based approach is proposed for monitoring and controlling the health constraints such as blood pressure (BP), haemoglobin (HB), sugar level in blood, and irregular cellular development. An IoT-based healthcare service is proposed to monitor the blood pressure, diabetes, and overweightness [91].

(3) Body Temperature Monitoring. Monitoring and measuring body temperature is an important part of healthcare applications. According to $\mathrm{m}$-IoT perception, the homeostasis maintenance varies according to the temperature of a body. The body monitoring sensors are fixed with the TelosBmote, and it gives precise and effective results in processing. The body temperature monitoring system is based on the home gateway over the IoT mechanism. It also assists in infrared detection and RFID module for the monitoring and measuring of body temperature [22].

(4) Oxygen Saturation Monitoring System. A measuring instrument "Pulse oximeter" is used for continuous monitoring of oxygen saturation in blood. The use of the IoT with pulse oximetry is beneficial for technology-based medical healthcare applications. A study of the CoAP-based healthcare system discusses the benefits of IoT-based pulse oximetry. A pulse oximeter Wrist OX2 device functionality is demonstrated by Nonin. This device has the ability of connectivity to Bluetooth and directly connects the sensor to the Monere platform. An IoT-based low-power and low-cost pulse oximeter device is proposed for monitoring remote patients. This device can continuously monitor patient's health through IoT network [2].

(5) ECG (Electrocardiogram) Monitoring System. ECG monitoring device has the capability to show the ECG waves to the user/patient. This device generates a specific medical report of a patient by collecting ECG bio-signals and uploads the data to the cloud network. It also provides an appropriate response to the user based on the collected data. IOIO-OTG microcontroller converts ECG signals to digital numbers by using the analog to digital converter, and then the generated binary file is uploaded to the cloud network for analysis and detection of abnormal health conditions. The main advantage of this equipment is to reduce waiting time in hospitals and emergency wards and to minimize visits to the health centres [48].

\subsubsection{Clustered Condition Applications}

(1) Wheelchair Management System. Keeping in view the health of the elder and disabled people, fully automated smart wheelchairs are also proposed by the researchers. In this field, IoT have the key role to expedite this work. The smart wheelchairs are equipped with various sensors which monitor the movement of the chair and also detect the status of the patient/user [91].

(2) Rehabilitation System. IoT can develop the rehabilitation system in terms of population growth-related problems and lack of health expertise. It can improve the abilities of the physical disable people. Body Sensor Network is proposed to enhance the rehabilitation system [91]. An ontology-based automating design determines that the IoT can be a more effective environment for providing actual information interactions. A number of IoT-based rehabilitation systems are as language training system for childhood, smart city medical rehabilitation system, and integrated application system for prisons [2].

(3) Healthcare Solutions Using Smartphones. At the present time, the smartphone has proved to control the electronic devices with sensors. In the field of healthcare, different apps for smartphones are provided to assist patients, furnish medical education, and provide initial training. Several software and hardware products are developed which expresses smartphone as a useful gadget in healthcare services. A list of the common smartphone apps for general healthcare is given in Table 7 [2].

3.4. RQ-4: What Are the Available Research Works in IoTfor Healthcare Using Mobile Computing? For years, a number of researchers and scholars are working to access data everywhere, process them on time, and wirelessly distribute in healthcare using IoT technology [111]. According to ABI Research, about 90 million wearable devices were used for healthcare, sport applications, and fitness in 2014. This number seems to be increased in the coming years [109]. Researchers performed a lot of work in the field of healthcare using Internet of Things, like in China, which is the most populated country, a system between the urban and rural healthcare, and insufficient information sharing is an obstacle in the healthcare service. To resolve such issues of distribution of data among hospitals, an integrated clinic information system has been designed to exchange the data among the health centres [111].

\subsubsection{Services of IoT in Healthcare}

(1) Ambient Assisted Living ( $A A L)$. In healthcare using Internet of things technology, Ambient Assisted Living (AAL) is designed to improve and facilitate the living of elders as well as the physically impaired people. The focus of researchers in the field of IoT in healthcare is to develop such intelligent system which can monitor and record daily activities of elders or disabled people residing in smart environment. The AAL system will bring an optimistic change on different aspects of health and lifestyle. The AAL system is based on multiple sensors for measuring body temperature, blood pressure, glucose, oxygen, and weight. This system is mostly adequate for the patients of diabetes and cardiac arrhythmia. Ambient Assisted Living systems allow doctors and family members to monitor their patient remotely [97]. Recent advancements in using IoT in healthcare helped the dream of AAL to become a reality [116].

(2) The Internet of $m$-health Things (m-IoT). The current advancement in ICT and IoT technology has brought it to 
TABLE 8: Detail of selected papers for RQ4.

\begin{tabular}{|c|c|c|c|c|}
\hline Ref\# & Method/Technique & Description & Goal/future work & $\begin{array}{l}\text { Pub. } \\
\text { year }\end{array}$ \\
\hline$[14]$ & Literature review & $\begin{array}{l}\text { This studies multimedia data processing in the } \\
\text { healthcare system based on IoT }\end{array}$ & $\begin{array}{l}\text { To secure healthcare frame by using blockchain } \\
\text { technology }\end{array}$ & 2019 \\
\hline [31] & Literature review & $\begin{array}{l}\text { It investigates the issue of maintaining medical } \\
\text { devices through data and monitoring }\end{array}$ & $\begin{array}{c}\text { To improve the reusability of medical devices } \\
\text { and maintenance }\end{array}$ & 2019 \\
\hline [28] & Survey/literature review & To build a system mode in the field of healthcare & $\begin{array}{l}\text { To achieve more target in EMS, to handle the } \\
\text { error of mindful-based reliability system in } \\
\text { healthcare }\end{array}$ & 2019 \\
\hline [22] & $\begin{array}{r}\text { Systematic } \\
\text { revie }\end{array}$ & $\begin{array}{c}\text { To know fog computing in the IoT healthcare } \\
\text { system }\end{array}$ & $\begin{array}{l}\text { To bring improvem } \\
\text { studies like low latenc }\end{array}$ & 2019 \\
\hline [111] & Literature review & $\begin{array}{l}\text { How to collect, access } \\
\text { data in medic }\end{array}$ & $\begin{array}{c}\text { To construct a UDA-IoT method for entire } \\
\text { business }\end{array}$ & 2014 \\
\hline [114] & & $\begin{array}{l}\text { The design, function, and } \\
\text { in the healthcar }\end{array}$ & $\begin{array}{c}\text { To implement WIoT in order to provide } \\
\text { services in healthcare with low cost and early } \\
\text { illness detection }\end{array}$ & 2013 \\
\hline$[100]$ & Experimental work & $\begin{array}{l}\text { A middleware base } \\
\text { data }\end{array}$ & $\begin{array}{l}\text { How M-hub could } \\
\text { scenarios like nu. }\end{array}$ & 2015 \\
\hline [119] & Experimental work & $\begin{array}{r}\text { m-Health based on IoT for c } \\
\text { observing fo }\end{array}$ & $\begin{array}{l}\text { To use m-health for food by children and to } \\
\text { work on NFC-enabled devices }\end{array}$ & 2012 \\
\hline [116] & Experimental work & $\begin{array}{l}\text { The rise in the use of AAL for } \\
\text { to know about AAL techni }\end{array}$ & To cope with challenges faced by AAL & 2012 \\
\hline [2] & Literature review & $\begin{array}{l}\text { The focus is to study IoT applications in } \\
\text { healthcare, to analyse the security \& privacy in } \\
\text { IoT, and to present a security model }\end{array}$ & $\begin{array}{l}\text { The IoT policies for the benefit of stakeholder in } \\
\text { the IoT-based healthcare system }\end{array}$ & 2015 \\
\hline [35] & Literature review & $\begin{array}{c}\text { To study about use of IoT in medical and smart } \\
\text { healthcare }\end{array}$ & $\begin{array}{c}\text { To study the security and privacy of } \mathrm{K} \text { - } \\
\text { healthcare }\end{array}$ & 2018 \\
\hline [20] & Literature review & $\begin{array}{l}\text { It describes how IoT-based healthcare solution } \\
\text { is implemented }\end{array}$ & $\begin{array}{c}\text { To get potential benefits from IoT healthcare } \\
\text { solution }\end{array}$ & 2019 \\
\hline [60] & Literature review & $\begin{array}{l}\text { To find and solve the issues of medical services } \\
\text { in intelligent community }\end{array}$ & $\begin{array}{c}\text { To develop a smart health community and to } \\
\text { enhance the medical services }\end{array}$ & 2018 \\
\hline [45] & $\begin{array}{l}\text { Systematic literature } \\
\text { review }\end{array}$ & $\begin{array}{c}\text { The research work is about how IoT and AI } \\
\text { applications are categorized }\end{array}$ & $\begin{array}{c}\text { The future work of the research paper can be } \\
\text { based upon adaptation of ecosystem technology } \\
\text { in different social, cultural, and technical } \\
\text { settings }\end{array}$ & 2018 \\
\hline [46] & Experiment research & $\begin{array}{l}\text { To present intelligent healthcare monitoring } \\
\text { system for human activity recognition by using } \\
\text { mining techniques }\end{array}$ & $\begin{array}{l}\text { To enable people to access the smart IoT, mobile } \\
\text { phone, and so on in the healthcare system }\end{array}$ & 2018 \\
\hline
\end{tabular}

the reality to monitor a patient remotely instead of diagnosing them physically in hospitals. A patient is equipped with certain sensors which get health data and transmit them to the datacentres through internet to keep them available for further decisions [94].

(3) Community Healthcare $(\mathrm{CH})$. The Community Healthcare $(\mathrm{CH})$ system is a network which covers a local area. The structure of a community healthcare seems as a "Virtual Health Centre." It is an IoT-based network, which monitors around the rural community or housing area [2].

(4) Children Health Information (CHI). The Children Health Information system is a platform for children to improve their health by changing their food habits when they are away from parents and eating outside from their homes. Children using a mobile device for tracking food get notification from their doctors, caretakers, or parents. Users can receive information regarding food through the augmented application. The health educator uses the stored information to provide feedback to the children and sends a notification to their parents. This enables parents to interact with their children to enhance family food habits [119]. The following data were analysed for the purpose of answering to RQ-4, as explained in Table 8 .

\section{Limitations}

The concept of IoT in the healthcare system with the support of mobile computing is good idea but IoT devices come up with very little processing speed and are not powerful due to limited processing functionalities. In the healthcare system, we need hard real-time transmission of data, any slight delay may lead to the fatal situation. The IoT devices have very little memory, so sometimes, the security protocols in the $\mathrm{m}$-health system with the support of IoT at both ends will be a challenging task to implement. The IoT devices are connected to internet, and these devices have the features of mobility; sometimes, change in platform or environment like operating system running on the smart phone may create the security compatibility issues due to rapid change in network configuration and setting. As in the IoT-based healthcare 
system, heterogeneous devices are used, so designing a topological structure, security model for multidevices, multiprotocol network, and cryptographic techniques to meet the requirements of all in IoT-based healthcare network in the context of mobile computing will be an issue.

\section{Conclusion and Future Lines of Research}

The discussion about using mobile computing in the IoT health-based system makes the healthcare system more fascinating by providing mobile-based services, computing applications, and user-friendly interfaces by which a user can easily record data about his or her health and can analyse them for different purposes.

The involvement of $\mathrm{m}$-health in IoT makes the healthcare system more compact by bringing various services, applications, third party APIs, and mobile sensors. The mobile computing can provide more privacy and security to the IoT devices that are vulnerable to any hacking attack. The mobile computing provides end-to-end security in order to secure the large amount of data that are transmitted to the IoT device to the server or from the server to IoT. The enormous applications such as glucose level sensing, oxygen saturation monitoring system, and body temperature monitoring of IoT with the support of mobile computing contribute towards bringing improvements in the healthcare system. The available research work in Internet of Healthcare Things is also getting popular with the concept of mobile computing technology.

(i) As with the advantages of IoT, some challenges also exist which need to be sort out. Some of the challenges for IoT devices along with the proposed solution are described here.

(ii) Data Management. As data management is a central aspect in the IoT environment, the interconnected objects generate and exchange massive amount of data which need to be stored and processed properly. It would be better if some enterprises provide enough storage to accommodate all the IoT data.

(iii) Privacy and Security. The IoT improves the productivity and enhances the quality of people life, and these improvements also increase the potential attacks and create a pathway for hackers. A recent research by Hewlett Packard in 2014 exposed that most of the IoT devices contain certain vulnerabilities due to insecure web interfaces, insufficient software protection, and authorization. A solution for such security challenges is to train developers and encourage users to use build-in security features of IoT devices.

(iv) Device Level Energy Issues. An important challenge in IoT devices is the continuous power supply to keep them interconnected. It should be known that the communication among the devices is mostly an energy-consuming task.

This research work presented a systematic literature review protocol to study how mobile computing assists IoT applications in healthcare, how mobile computing contributes to the current and future research work of IoT in the healthcare system, brings privacy and security in health IoT devices, and affects the IoT in the healthcare system. The intentions of the paper are to study the impacts of mobile computing on IoT in healthcare environment or smart hospitals in light of our systematic literature review protocol. The proposed study reports the papers that were included based on filtering process by title, abstract, and contents, and a total of 116 primary studies were included to support the proposed research. These papers were then analysed, and the quality assessments of these selected papers were done for research questions defined. The results of the research reveal that there is an increase of research work done in the field of IoT using mobile computing.

\section{Conflicts of Interest}

The authors declare that there are no conflicts of interest regarding this paper.

\section{References}

[1] S. Tyagi, A. Agarwal, and P. Maheshwari, "A conceptual framework for IoT-based healthcare system using cloud computing," in Proceedings of the 2016 6th International Conference-Cloud System and Big Data Engineering (Confluence), pp. 503-507, Noida, India, January 2016.

[2] S. M. R. Islam, D. Kwak, M. H. Kabir, M. Hossain, and K.-S. Kwak, "The Internet of Things for health care: a comprehensive survey," IEEE Access, vol. 3, pp. 678-708, 2015.

[3] Y. Yuehong, Y. Zeng, X. Chen, and Y. Fan, "The Internet of Things in healthcare: an overview," Journal of Industrial Information Integration, vol. 1, pp. 3-13, 2016.

[4] S. H. Almotiri, M. A. Khan, and M. A. Alghamdi, "Mobile health (m-health) system in the context of IoT," in Proceedings of the 2016 IEEE 4th International Conference on Future Internet of Things and Cloud Workshops (FiCloudW), pp. 39-42, Vienna, Austria, August 2016.

[5] Y. Karaca, M. Moonis, Y.-D. Zhang, and C. Gezgez, "Mobile cloud computing based stroke healthcare system," International Journal of Information Management, vol. 45, pp. 250-261, 2019.

[6] B. Kitchenham and S. Charters, "Guidelines for performing systematic literature reviews in software engineering," Tech. Rep. EBSE-2007-01, Software Engineering Group School of Computer Science and Mathematics, Keele University Keele, Staffs ST5 5BG, UK, 2007.

[7] S. Asadi, R. Abdullah, Y. Yah, and S. Nazir, "Understanding institutional repository in higher learning institutions: a systematic literature review and directions for future research," IEEE Access, vol. 7, pp. 35242-35263, 2019.

[8] S. Nazir, S. Shahzad, and N. Mukhtar, "Software birthmark design and estimation: a systematic literature review," Arabian Journal for Science and Engineering, vol. 44, no. 4, pp. 3905-3927, 2019.

[9] C. Wohlin, "Guidelines for snowballing in systematic literature studies and a replication in software engineering," in Proceedings of the 18th International Conference on Evaluation and Assessment in Software Engineering, Berlin, Germany, March 2014. 
[10] P. Kaur, R. Kumar, and M. Kumar, "A healthcare monitoring system using random forest and Internet of Things (IoT)," Multimedia Tools and Applications, vol. 78, no. 14, pp. 19905-19916, 2019.

[11] S. Mohapatra, S. Mohanty, and S. Mohanty, "Smart healthcare: an approach for ubiquitous healthcare management using IoT," in Big Data Analytics for Intelligent Healthcare Management, pp. 175-196, Elsevier, Amsterdam, Netherlands, 2019.

[12] A. Dumka and A. Sah, "Smart ambulance system using concept of big data and Internet of Things," in Healthcare Data Analytics and Management, pp. 155-176, Elsevier, Amsterdam, Netherlands, 2019.

[13] P. K. D. Pramanik, B. K. Upadhyaya, S. Pal, and T. Pal, "Internet of Things, smart sensors, and pervasive systems: enabling connected and pervasive healthcare," in Healthcare Data Analytics and Management, pp. 1-58, Elsevier, Amsterdam, Netherlands, 2019.

[14] G. Rathee, A. Sharma, H. Saini, R. Kumar, and R. Iqbal, “A hybrid framework for multimedia data processing in IoThealthcare using blockchain technology," Multimedia Tools and Applications, vol. 78, pp. 1-23, 2019.

[15] H. Hamidi, "An approach to develop the smart health using Internet of Things and authentication based on biometric technology," Future Generation Computer Systems, vol. 91, pp. 434-449, 2019.

[16] A. Arfaoui, A. Kribeche, and S.-M. Senouci, "Context-aware anonymous authentication protocols in the Internet of Things dedicated to e-health applications," Computer Networks, vol. 159, pp. 23-36, 2019.

[17] P. P. Ray, D. Dash, and D. De, "Edge computing for Internet of Things: a survey, e-healthcare case study and future direction," Journal of Network and Computer Applications, vol. 140, pp. 1-22, 2019.

[18] M. G. R. Alam, M. S. Munir, M. Z. Uddin, M. S. Alam, T. N. Dang, and C. S. Hong, "Edge-of-things computing framework for cost-effective provisioning of healthcare data," Journal of Parallel and Distributed Computing, vol. 123, pp. 54-60, 2019.

[19] A. A. Mutlag, M. K. A. Ghani, N. Arunkumar, M. A. Mohammed, and O. Mohd, "Enabling technologies for fog computing in healthcare IoT systems," Future Generation Computer Systems, vol. 90, pp. 62-78, 2019.

[20] S. Zeadally and O. Bello, "Harnessing the power of Internet of Things based connectivity to improve healthcare," Internet of Things, Article ID 100074, 2019.

[21] H. Elazhary, "Internet of Things (IoT), mobile cloud, cloudlet, mobile IoT, IoT cloud, fog, mobile edge, and edge emerging computing paradigms: disambiguation and research directions," Journal of Network and Computer Applications, vol. 128, pp. 108-140, 2018.

[22] M. M. Dhanvijay and S. C. Patil, "Internet of Things: a survey of enabling technologies in healthcare and its applications," Computer Networks, vol. 153, pp. 113-131, 2019.

[23] A. H. Sodhro, Z. Luo, A. K. Sangaiah, and S. W. Baik, "Mobile edge computing based QoS optimization in medical healthcare applications," International Journal of Information Management, vol. 45, pp. 308-318, 2019.

[24] T. Saheb and L. Izadi, "Paradigm of IoT big data analytics in healthcare industry: a review of scientific literature and mapping of research trends," Telematics and Informatics, vol. 41, pp. 70-85, 2019.

[25] S. Pal, M. Hitchens, V. Varadharajan, and T. Rabehaja, "Policy-based access control for constrained healthcare resources," in Proceedings of the 2018 IEEE 19th International Symposium on "A World of Wireless, Mobile and Multimedia Networks” (WoWMoM), pp. 588-599, Chania, Greece, June 2018.

[26] A. Onasanya and M. Elshakankiri, "Smart integrated IoT healthcare system for cancer care," Wireless Networks, vol. 25, no. 165, pp. 1-16, 2019.

[27] M.-H. Maras and A. S. Wandt, "Enabling mass surveillance: data aggregation in the age of big data and the Internet of Things," Journal of Cyber Policy, vol. 4, no. 2, pp. 160-177, 2019.

[28] M. N. D. Tuan, N. N. Thanh, and L. L. Tuan, “Applying a mindfulness-based reliability strategy to the Internet of Things in healthcare-A business model in the Vietnamese market," Technological Forecasting and Social Change, vol. 140, pp. 54-68, 2019.

[29] Akshat, Gaurav, Zahid et al., "A smart healthcare monitoring system using smartphone interface," in Proceedings of the 2018 4th International Conference on Devices, Circuits and Systems (ICDCS), pp. 228-231, Coimbatore, India, March 2018.

[30] V. Jagadeeswari, V. Subramaniyaswamy, R. Logesh, and V. Vijayakumar, "A study on medical Internet of Things and big data in personalized healthcare system," Health Information Science and Systems, vol. 6, no. 1, p. 14, 2018.

[31] J. Maktoubian and K. Ansari, "An IoT architecture for preventive maintenance of medical devices in healthcare organizations," Health and Technology, vol. 9, no. 3, pp. 233-243, 2019.

[32] V. Sindhura, P. Ramya, and S. Yelisetti, "An IOT based smart mobile health monitoring system," in Proceedings of the 2018 Second International Conference on Inventive Communication and Computational Technologies (ICICCT), pp. 11861192, Coimbatore, India, April 2018.

[33] S. Zemmoudj, N. Bermad, and M. Omar, "Context-aware pseudonymization and authorization model for IoT-based smart hospitals," Journal of Ambient Intelligence and Humanized Computing, vol. 10, no. 11, pp. 4473-4490, 2019.

[34] A. A. Abdellatif, M. G. Khafagy, A. Mohamed, and C.-F. Chiasserini, "EEG-based transceiver design with data decomposition for healthcare IoT applications," IEEE Internet of Things Journal, vol. 5, no. 5, pp. 3569-3579, 2018.

[35] R. Rashid and M. A. Shah, "EK-Healthcare: Effectiveness of IoT in the medical field with enhanced features," in Proceedings of the 2018 24th International Conference on Automation and Computing (ICAC), pp. 1-6, Newcastle upon Tyne, UK, September 2018.

[36] M. Bhatia and S. K. Sood, "Exploring temporal analytics in fog-cloud architecture for smart office healthcare," Mobile Networks and Applications, vol. 24, no. 4, pp. 1392-1410, 2019.

[37] P. Verma and S. K. Sood, "Fog assisted-IoT enabled patient health monitoring in smart homes," IEEE Internet of Things Journal, vol. 5, no. 3, pp. 1789-1796, 2018.

[38] S. A. Khowaja, A. G. Prabono, F. Setiawan, B. N. Yahya, and S.-L. Lee, "Contextual activity based Healthcare Internet of Things, Services, and People (HIoTSP): an architectural framework for healthcare monitoring using wearable sensors," Computer Networks, vol. 145, pp. 190-206, 2018.

[39] A. Kumari, S. Tanwar, S. Tyagi, and N. Kumar, "Fog computing for healthcare 4.0 environment: opportunities and challenges," Computers \& Electrical Engineering, vol. 72, pp. 1-13, 2018. 
[40] S. Chatterjee, J. Byun, K. Dutta, R. U. Pedersen, A. Pottathil, and H. Xie, "Designing an Internet-of-Things (IoT) and sensor-based in-home monitoring system for assisting diabetes patients: iterative learning from two case studies," European Journal of Information Systems, vol. 27, no. 6, pp. 670-685, 2018.

[41] M. Pasha and S. M. W. Shah, "Framework for E-Health systems in IoT-based environments," Wireless Communications and Mobile Computing, vol. 2018, Article ID 6183732, 11 pages, 2018.

[42] N. Koutsouris, P. Kosmides, K. Demestichas, E. Adamopoulou, K. Giannakopoulou, and V. De Luca, "InLife: a platform enabling the exploitation of IoT and gamification in healthcare," in Proceedings of the 2018 14th International Conference on Wireless and Mobile Computing, Networking and Communications (WiMob), pp. 224-230, Limassol, Cyprus, October 2018.

[43] T. Sigwele, Y. F. Hu, M. Ali, J. Hou, M. Susanto, and H. Fitriawan, "Intelligent and energy efficient mobile smartphone gateway for healthcare smart devices based on 5G," in Proceedings of the 2018 IEEE Global Communications Conference (GLOBECOM), pp. 1-7, Abu Dhabi, UAE, December 2018.

[44] X. Ma, Z. Wang, S. Zhou, H. Wen, and Y. Zhang, "Intelligent healthcare systems assisted by data analytics and mobile computing," Wireless Communications and Mobile Computing, vol. 2018, Article ID 3928080, 16 pages, 2018.

[45] R. Shah and A. Chircu, "IOT and AI IN healthcare: a systematic literature review," Issues in Information Systems, vol. 19, 2018.

[46] A. Subasi, M. Radhwan, R. Kurdi, and K. Khateeb, "IoT based mobile healthcare system for human activity recognition," in Proceedings of the 2018 15th Learning and Technology Conference (L\&T), pp. 29-34, Jeddah, Saudi Arabia, Febuary 2018.

[47] G. Yang, M. Jiang, W. Ouyang et al., "IoT-based remote pain monitoring system: from device to cloud platform," IEEE Journal of Biomedical and Health Informatics, vol. 22, no. 6, pp. 1711-1719, 2018.

[48] H. Ahmadi, G. Arji, L. Shahmoradi, R. Safdari, M. Nilashi, and M. Alizadeh, "The application of Internet of Things in healthcare: a systematic literature review and classification," Universal Access in the Information Society, vol. 18, no. 4, pp. 837-869, 2019.

[49] M. M. E. Mahmoud, J. J. P. C. Rodrigues, K. Saleem, J. AlMuhtadi, N. Kumar, and V. Korotaev, "Towards energyaware fog-enabled cloud of things for healthcare," Computers \& Electrical Engineering, vol. 67, pp. 58-69, 2018.

[50] F. T. Jaigirdar, "Trust based security solution for Internet of Things healthcare solution: an end-to-end trustworthy architecture," in Proceedings of the 2018 ACM International Joint Conference and 2018 International Symposium on Pervasive and Ubiquitous Computing and Wearable Computers, pp. 1757-1760, Singapore, October 2018.

[51] O. Geman, I. Chiuchisan, I. Ungurean, M. Hagan, and M. Arif, "Ubiquitous healthcare system based on the sensors network and android Internet of Things gateway," in Proceedings of the 2018 IEEE SmartWorld, Ubiquitous Intelligence \& Computing, Advanced \& Trusted Computing, Scalable Computing \& Communications, Cloud \& Big Data Computing, Internet of People and Smart City Innovation (SmartWorld/SCALCOM/UIC/ ATC/CBDCom/IOP/SCI), pp. 1390-1395, Guangzhou, China, October 2018.

[52] F. Nausheen and S. H. Begum, "Healthcare IoT: benefits, vulnerabilities and solutions," in Proceedings of the 2018 2nd
International Conference on Inventive Systems and Control (ICISC), pp. 517-522, Coimbatore, India, January 2018.

[53] A. Papageorgiou, M. Strigkos, E. Politou, E. Alepis, A. Solanas, and C. Patsakis, "Security and privacy analysis of mobile health applications: the alarming state of practice," IEEE Access, vol. 6, pp. 9390-9403, 2018.

[54] A. Chacko and T. Hayajneh, "Security and privacy issues with IoT in healthcare," EAI Endorsed Transactions on Pervasive Health and Technology, vol. 4, no. 1, Article ID 155079, 2018.

[55] A. Ullah, I. Sehr, M. Akbar, and H. Ning, "FoG assisted secure de-duplicated data dissemination in smart healthcare IoT," in Proceedings of the 2018 IEEE International Conference on Smart Internet of Things (SmartIoT), pp. 166-171, Xi'an, China, August 2018.

[56] W. Sun, Z. Cai, Y. Li, F. Liu, S. Fang, and G. Wang, "Security and privacy in the medical Internet of Things: a review," Security and Communication Networks, vol. 2018, Article ID 5978636, 9 pages, 2018.

[57] P. Pawar and A. Trivedi, "Device-to-device communication based IoT system: benefits and challenges," IETE Technical Review, vol. 36, no. 4, pp. 362-374, 2018.

[58] A. Godfrey, V. Hetherington, H. Shum, P. Bonato, N. H. Lovell, and S. Stuart, "From A to Z: wearable technology explained," Maturitas, vol. 113, pp. 40-47, 2018.

[59] A. Paul, H. Pinjari, W.-H. Hong, H. C. Seo, and S. Rho, "Fog computing-based IoT for health monitoring system," Journal of Sensors, vol. 2018, Article ID 1386470, 7 pages, 2018.

[60] Y. Wang, J. He, H. Zhao, Y.-H. Han, and X.-J. Huang, "Intelligent community medical service based on Internet of Things," Journal of Interdisciplinary Mathematics, vol. 21, no. 5, pp. 1121-1126, 2018.

[61] A. Shahzad, Y. S. Lee, M. Lee, Y.-G. Kim, and N. Xiong, "Realtime cloud-based health tracking and monitoring system in designed boundary for cardiology patients," Journal of Sensors, vol. 2018, Article ID 3202787, 15 pages, 2018.

[62] C. A. da Costa, C. F. Pasluosta, B. Eskofier, D. B. da Silva, and R. da Rosa Righi, "Internet of Health Things: toward intelligent vital signs monitoring in hospital wards," Artificial intelligence in medicine, vol. 89, pp. 61-69, 2018.

[63] A. M. Elmisery, S. Rho, and M. Aborizka, "A new computing environment for collective privacy protection from constrained healthcare devices to IoT cloud services," Cluster Computing, vol. 22, no. 1, pp. 1611-1638, 2019.

[64] K. Kadarla, S. Sharma, T. Bhardwaj, and A. Chaudhary, "A simulation study of response times in cloud environment for IoT-based healthcare workloads," in Proceedings of the 2017 IEEE 14th International Conference on Mobile Ad Hoc and Sensor Systems (MASS), pp. 678-683, Orlando, FL, USA, October 2017.

[65] E. Gonzalez, R. Peña, A. Avila, C. Vargas-Rosales, and D. Munoz-Rodriguez, "A systematic review on recent advances in mhealth systems: deployment architecture for emergency response," Journal of Healthcare Engineering, vol. 2017, Article ID 9186270, 13 pages, 2017.

[66] J. Hanen, Z. Kechaou, and M. B. Ayed, "Agent technology based modelization systems for healthcare," IETE Journal of Research, vol. 63, no. 5, pp. 630-642, 2017.

[67] J. Qi, P. Yang, G. Min, O. Amft, F. Dong, and L. Xu, "Advanced Internet of Things for personalised healthcare systems: a survey," Pervasive and Mobile Computing, vol. 41, pp. 132-149, 2017.

[68] Z. U. Abideen and M. A. Shah, "An IoT based robust healthcare model for continuous health monitoring," in 
Proceedings of the 2017 23rd International Conference on Automation and Computing (ICAC), pp. 1-6, Huddersfield, UK, September 2017.

[69] P. Verma, S. K. Sood, and S. Kalra, "Cloud-centric IoT based student healthcare monitoring framework," Journal of Ambient Intelligence and Humanized Computing, vol. 9, no. 5, pp. 1293-1309, 2018.

[70] D. A. M. Budida and R. S. Mangrulkar, "Design and implementation of smart healthcare system using IoT," in Proceedings of the 2017 International Conference on Innovations in Information, Embedded and Communication Systems (ICIIECS), pp. 1-7, Coimbatore, India, March 2017.

[71] J. J. P. C. Rodrigues, D. B. D. R. Segundo, H. A. Junqueira et al., "Enabling technologies for the Internet of Health Things," IEEE Access, vol. 6, pp. 13129-13141, 2018.

[72] S. A. Bharadwaj, D. Yarravarapu, S. C. K. Reddy, T. Prudhvi, K. Sandeep, and O. S. D. Reddy, "Enhancing healthcare using $\mathrm{m}$-care box (monitoring non-compliance of medication)," in Proceedings of the 2017 International Conference on I-SMAC (IoT in Social, Mobile, Analytics and Cloud)(I-SMAC), pp. 352-356, Tirupur, India, Febuary 2017.

[73] A. I. E. S. Eldein, H. H. Ammar, and D. G. Dzielski, "Enterprise architecture of mobile healthcare for large crowd events," in Proceedings of the 2017 6th International Conference on Information and Communication Technology and Accessibility (ICTA), pp. 1-6, Hammamet, Tunisia, December 2017.

[74] S. F. Khan, "Health care monitoring system in Internet of Things (IoT) by using RFID," in Proceedings of the 2017 6th International Conference on Industrial Technology and Management (ICITM), pp. 198-204, Cambridge, UK, March 2017.

[75] A. Rashed, A. Ibrahim, A. Adel et al., "Integrated IoT medical platform for remote healthcare and assisted living," in Proceedings of the 2017 Japan-Africa Conference on Electronics, Communications and Computers (JAC-ECC), pp. 160-163, Alexandria, Egypt, December 2017.

[76] S. Chaudhury, D. Paul, S. Haldar, R. Mukherjee, and S. Haldar, "Internet of Thing based healthcare monitoring system," in Proceedings of the 2017 8th IEEE Annual Information Technology, Electronics and Mobile Communication Conference (IEMCON), pp. 531-535, Bangkok, Thailand, August 2017.

[77] P. Singh and A. Jasuja, "IoT based low-cost distant patient ECG monitoring system," in Proceedings of the 2017 International Conference on Computing, Communication and Automation (ICCCA), pp. 1330-1334, Greater Noida, India, May 2017.

[78] M. Bansal and B. Gandhi, "IoT based smart health care system using CNT electrodes (for continuous ECG monitoring)," in Proceedings of the 2017 International Conference on Computing, Communication and Automation (ICCCA), pp. 1324-1329, Greater Noida, India, May 2017.

[79] K. Michalakis and G. Caridakis, "IoT interface for healthcare applications," in Proceedings of the 10th International Conference on PErvasive Technologies Related to Assistive Environments, pp. 232-233, Island of Rhodes, Greece, June 2017.

[80] B. Mittelstadt, "Ethics of the health-related Internet of Things: a narrative review," Ethics and Information Technology, vol. 19, no. 3, pp. 157-175, 2017.

[81] S. Pal, M. Hitchens, V. Varadharajan, and T. Rabehaja, "On design of a fine-grained access control architecture for securing IoT-enabled smart healthcare systems," in Proceedings of the 14th EAI International Conference on Mobile and Ubiquitous Systems: Computing, Networking and Services, pp. 432-441, Melbourne, Australia, November 2017.

[82] J.-X. Hu, C.-L. Chen, C.-L. Fan, and K.-h. Wang, "An intelligent and secure health monitoring scheme using IoT sensor based on cloud computing," Journal of Sensors, vol. 2017, Article ID 3734764, 11 pages, 2017.

[83] A. Darwish, A. E. Hassanien, M. Elhoseny, A. K. Sangaiah, and K. Muhammad, "The impact of the hybrid platform of Internet of Things and cloud computing on healthcare systems: opportunities, challenges, and open problems," Journal of Ambient Intelligence and Humanized Computing, vol. 10, no. 10, pp. 4151-4166, 2019.

[84] P. Binu, K. Thomas, and N. P. Varghese, "Highly secure and efficient architectural model for IoT based health care systems," in Proceedings of the 2017 International Conference on Advances in Computing, Communications and Informatics (ICACCI), pp. 487-493, Manipal, India, September 2017.

[85] P. Sethi and S. R. Sarangi, "Internet of Things: architectures, protocols, and applications," Journal of Electrical and Computer Engineering, vol. 2017, Article ID 9324035, 25 pages, 2017.

[86] S. Jabbar, F. Ullah, S. Khalid, M. Khan, and K. Han, "Semantic interoperability in heterogeneous IoT infrastructure for healthcare," Wireless Communications and Mobile Computing, vol. 2017, Article ID 9731806, 10 pages, 2017.

[87] D. Korzun, "Internet of Things meets mobile health systems in smart spaces: an overview," in Internet of Things and Big Data Technologies for Next Generation Healthcare, pp. 111-129, Springer, Berlin, Germany, 2017.

[88] A. M. Elmisery, S. Rho, and D. Botvich, "A fog based middleware for automated compliance with OECD privacy principles in internet of healthcare things," IEEE Access, vol. 4, pp. 8418-8441, 2016.

[89] P. K. Gupta, B. T. Maharaj, and R. Malekian, "A novel and secure IoT based cloud centric architecture to perform predictive analysis of users activities in sustainable health centres," Multimedia Tools and Applications, vol. 76, no. 18, pp. 18489-18512, 2017.

[90] M. K. Kim, H. Ter Jung, S. D. Kim, and H. J. La, “A personal health index system with IoT devices," in Proceedings of the 2016 IEEE International Conference on Mobile Services (MS), pp. 174-177, San Francisco, CA, USA, June 2016.

[91] A. B. Pawar and S. Ghumbre, "A survey on IoT applications, security challenges and counter measures," in Proceedings of the 2016 International Conference on Computing, Analytics and Security Trends (CAST), pp. 294-299, Pune, India, December 2016.

[92] Z. Yang, Q. Zhou, L. Lei, K. Zheng, and W. Xiang, "An IoTcloud based wearable ECG monitoring system for smart healthcare," Journal of Medical Systems, vol. 40, no. 12, p. 286, 2016.

[93] J. Chaiwongsai, P. Preecha, and S. Intem, "Automated patient appointment reminder for cross-platform mobile application," in Proceedings of the 2016 International Symposium on Intelligent Signal Processing and Communication Systems (ISPACS), pp. 1-6, Phuket, Thailand, October 2016.

[94] K. Ullah, M. A. Shah, and S. Zhang, "Effective ways to use Internet of Things in the field of medical and smart health care," in Proceedings of the 2016 International Conference on Intelligent Systems Engineering (ICISE), pp. 372-379, Islamabad, Pakistan, January 2016.

[95] D. T. Lai, "Keynote talk: harnessing health IOT for smart healthcare," in Proceedings of the First Workshop on IoT- 
Enabled Healthcare and Wellness Technologies and Systems, p. 1, Singapore, June 2016.

[96] J. Gómez, B. Oviedo, and E. Zhuma, "Patient monitoring system based on Internet of Things," Procedia Computer Science, vol. 83, pp. 90-97, 2016.

[97] B. d. T. P. Gomes, L. C. M. Muniz, F. J. S. e Silva, L. E. T. Ríos, and M. Endler, "A comprehensive and scalable middleware for ambient assisted living based on cloud computing and Internet of Things," Concurrency and Computation: Practice and Experience, vol. 29, no. 11, p. e4043, 2017.

[98] A. P. Plageras, K. E. Psannis, Y. Ishibashi, and B.-G. Kim, "IoT-based surveillance system for ubiquitous healthcare," in Proceedings of the IECON 2016-42nd Annual Conference of the IEEE Industrial Electronics Society, pp. 6226-6230, Florence, Italy, October 2016.

[99] A. Ukil, S. Bandyoapdhyay, C. Puri, and A. Pal, "IoT healthcare analytics: the importance of anomaly detection," in Proceedings of the 2016 IEEE 30th International Conference on Advanced Information Networking and Applications (AINA), pp. 994-997, Crans-Montana, Switzerland, March 2016.

[100] P. Yang, M. Hanneghan, J. Qi, Z. Deng, F. Dong, and D. Fan, "Improving the validity of lifelogging physical activity measures in an Internet of Things environment," in Proceedings of the 2015 IEEE International Conference on Computer and Information Technology; Ubiquitous Computing and Communications; Dependable, Autonomic and Secure Computing; Pervasive Intelligence and Computing, pp. 2309-2314, Liverpool, UK, October 2015.

[101] N. A. Ali and M. Abu-Elkheir, "Internet of nano-things healthcare applications: requirements, opportunities, and challenges," in Proceedings of the 2015 IEEE 11th International Conference on Wireless and Mobile Computing, Networking and Communications (WiMob), pp. 9-14, Abu Dhabi, UAE, October 2015.

[102] Y. E. Gelogo, J.-w. Oh, J. W. Park, and H.-K. Kim, "Internet of Things (IoT) driven U-healthcare system architecture," in Proceedings of the 2015 8th International Conference on BioScience and Bio-Technology (BSBT), pp. 24-26, Jeju Island, South Korea, November 2015.

[103] D. Saxena, V. Raychoudhury, and N. SriMahathi, "SmartHealth-NDNoT: named data network of things for healthcare services," in Proceedings of the 2015 Workshop on Pervasive Wireless Healthcare-MobileHealth'15, pp. 45-50, Hangzhou, China, June 2015.

[104] B. Martínez-Pérez, I. De La Torre-Díez, and M. LópezCoronado, "Privacy and security in mobile health apps: a review and recommendations," Journal of Medical Systems, IEEE, vol. 39, no. 1, p. 181, 2015.

[105] R. K. Kodali, G. Swamy, and B. Lakshmi, "An implementation of IoT for healthcare," in Proceedings of the 2015 IEEE Recent Advances in Intelligent Computational Systems (RAICS), pp. 411-416, Trivandrum, India, December 2015.

[106] M. Thangaraj, P. P. Ponmalar, and S. Anuradha, "Internet of Things (IOT) enabled smart autonomous hospital management system-A real world health care use case with the technology drivers," in Proceedings of the 2015 IEEE International Conference on Computational Intelligence and Computing Research (ICCIC), pp. 1-8, Madurai, India, December 2015.

[107] J. Mohammed, C.-H. Lung, A. Ocneanu, A. Thakral, C. Jones, and A. Adler, "Internet of Things: remote patient monitoring using web services and cloud computing," in Proceedings of the 2014 IEEE International Conference on
Internet of Things (iThings), and IEEE Green Computing and Communications (GreenCom) and IEEE Cyber, Physical and Social Computing (CPSCom), pp. 256-263, Taipei, Taiwan, September 2014.

[108] F. Fernandez and G. C. Pallis, "Opportunities and challenges of the Internet of Things for healthcare: systems engineering perspective," in Proceedings of the 2014 4th International Conference on Wireless Mobile Communication and Healthcare-Transforming Healthcare through Innovations in Mobile and Wireless Technologies (MOBIHEALTH), pp. 263-266, Athens, Greece, 2014.

[109] S. Hiremath, G. Yang, and K. Mankodiya, "Wearable Internet of Things: concept, architectural components and promises for person-centered healthcare," in Proceedings of the 2014 4th International Conference on Wireless Mobile Communication and Healthcare-Transforming Healthcare through Innovations in Mobile and Wireless Technologies (MOBIHEALTH), pp. 304-307, Athens, Greece, 2014.

[110] Z. Pang and J. Tian, "Ecosystem-driven design of in-home terminals based on open platform for the Internet-ofThings," in Proceedings of the 16th International Conference on Advanced Communication Technology, pp. 369-377, PyeongChang, South Korea, 2014.

[111] B. Xu, L. D. Xu, H. Cai, C. Xie, J. Hu, and F. Bu, "Ubiquitous data accessing method in IoT-based information system for emergency medical services," IEEE Transactions on Industrial Informatics, vol. 10, no. 2, pp. 1578-1586, 2014.

[112] A. J. Jara, M. A. Zamora, and A. F. Skarmeta, "Drug identification and interaction checker based on IoT to minimize adverse drug reactions and improve drug compliance," Personal and Ubiquitous Computing, vol. 18, no. 1, pp. 5-17, 2014.

[113] Z. Pang, Q. Chen, J. Tian, L. Zheng, and E. Dubrova, "Ecosystem analysis in the design of open platform-based inhome healthcare terminals towards the internet-of-things," in Proceedings of the 2013 15th International Conference on Advanced Communications Technology (ICACT), pp. 529534, PyeongChang, South Korea, 2013.

[114] K. Kai, Z.-b. Pang, and W. Cong, "Security and privacy mechanism for health Internet of Things," The Journal of China Universities of Posts and Telecommunications, vol. 20, pp. 64-68, 2013.

[115] N. Alharbe, A. S. Atkins, and A. S. Akbari, "Application of ZigBee and RFID technologies in healthcare in conjunction with the Internet of Things," in Proceedings of International Conference on Advances in Mobile Computing \& Multimedia, p. 191, Vienna, Austria, December 2013.

[116] P. Rashidi and A. Mihailidis, "A survey on ambient-assisted living tools for older adults," IEEE Journal of Biomedical and Health Informatics, vol. 17, no. 3, pp. 579-590, 2012.

[117] C. Doukas and I. Maglogiannis, "Bringing IoT and cloud computing towards pervasive healthcare," in Proceedings of the 2012 Sixth International Conference on Innovative Mobile and Internet Services in Ubiquitous Computing, pp. 922-926, Palermo, Italy, July 2012.

[118] W. AL-Mawee, "Privacy and security issues in IoT healthcare applications for the disabled users a survey," Master thesis, Western Michigan University, Kalamazoo, MI, USA, 2012.

[119] M. Vazquez-Briseno, C. Navarro-Cota, J. I. Nieto-Hipolito, E. Jimenez-Garcia, and J. Sanchez-Lopez, "A proposal for using the Internet of Things concept to increase children's health awareness," in Proceedings of the CONIELECOMP 2012, 22nd International Conference on Electrical 
Communications and Computers, pp. 168-172, Cholula, Puebla, Mexico, February 2012.

[120] R. S. H. Istepanian, S. Hu, N. Y. Philip, and A. Sungoor, "The potential of Internet of $\mathrm{m}$-health Things " $\mathrm{m}$-IoT" for noninvasive glucose level sensing," in Proceedings of the 2011 Annual International Conference of the IEEE Engineering in Medicine and Biology Society, pp. 5264-5266, Boston, MA, USA, August 2011.

[121] J. M. Norris and L. Ortega, "Effectiveness of L2 instruction: a research synthesis and quantitative meta-analysis," Language Learning, vol. 50, no. 3, pp. 417-528, 2000. 


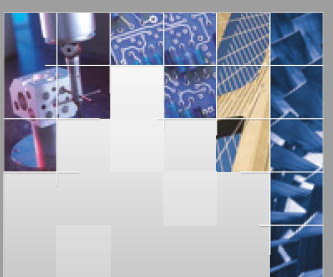

\section{Enfincering}
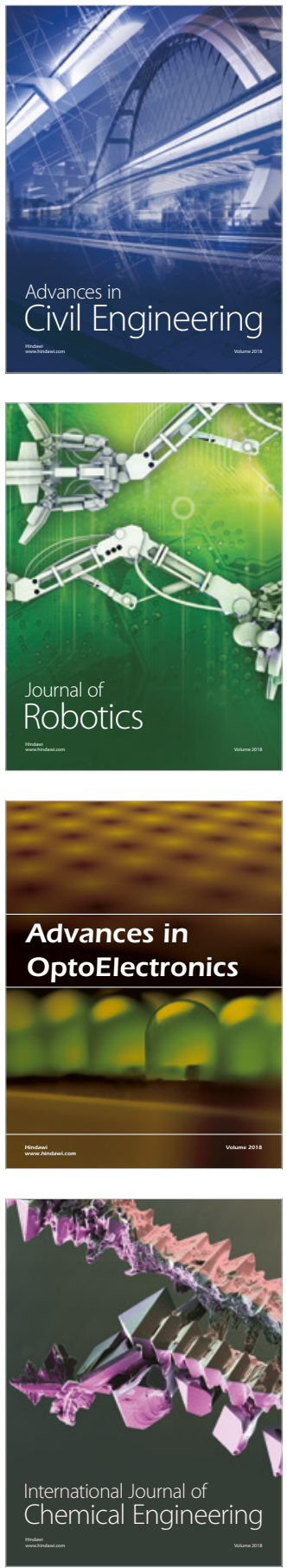

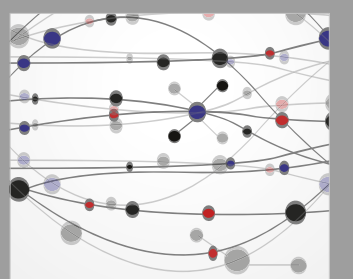

\section{Rotating \\ Machinery}

The Scientific World Journal

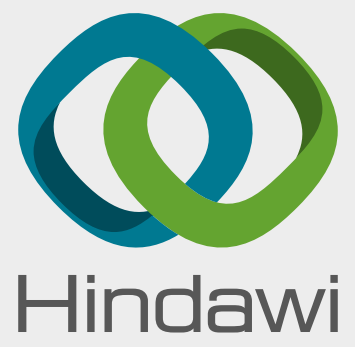

Submit your manuscripts at

www.hindawi.com
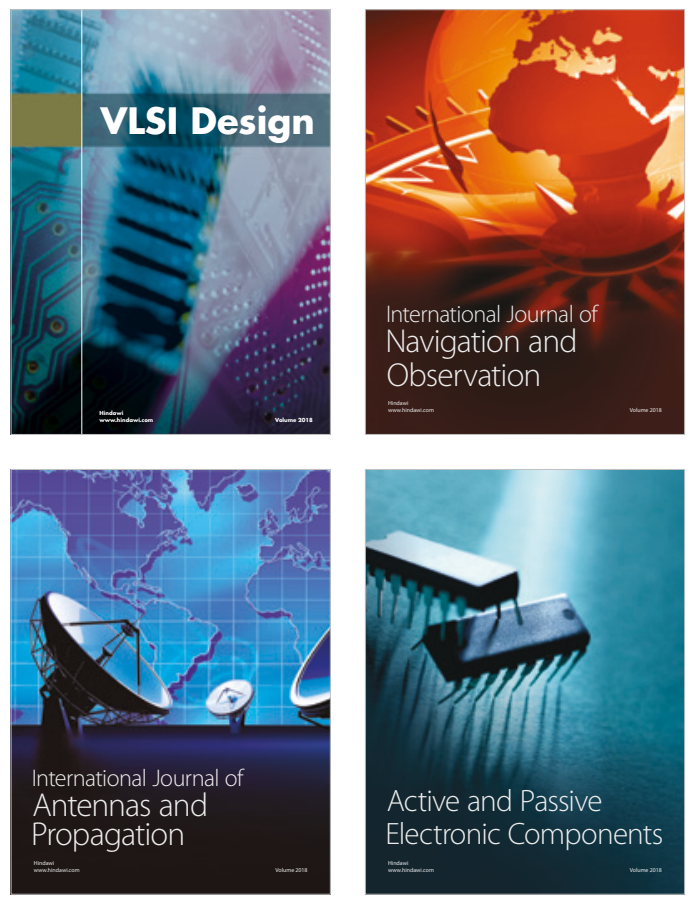
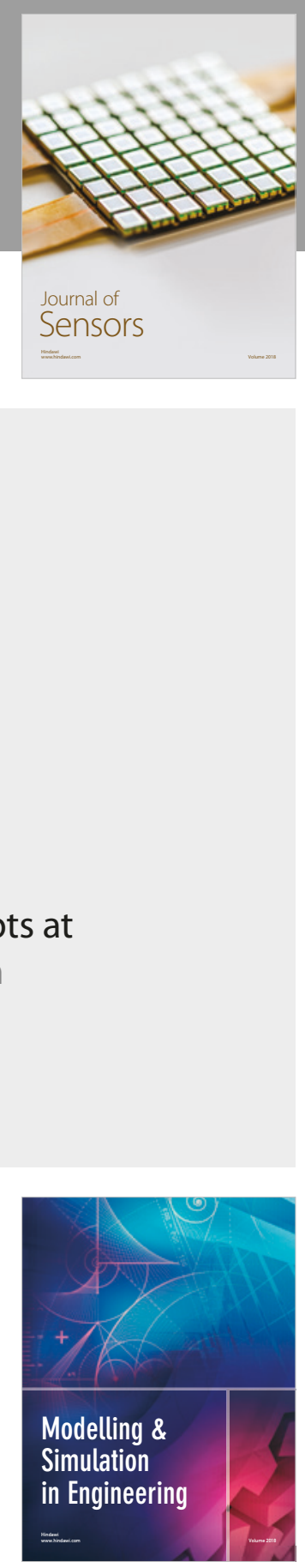

\section{Advances \\ Multimedia}
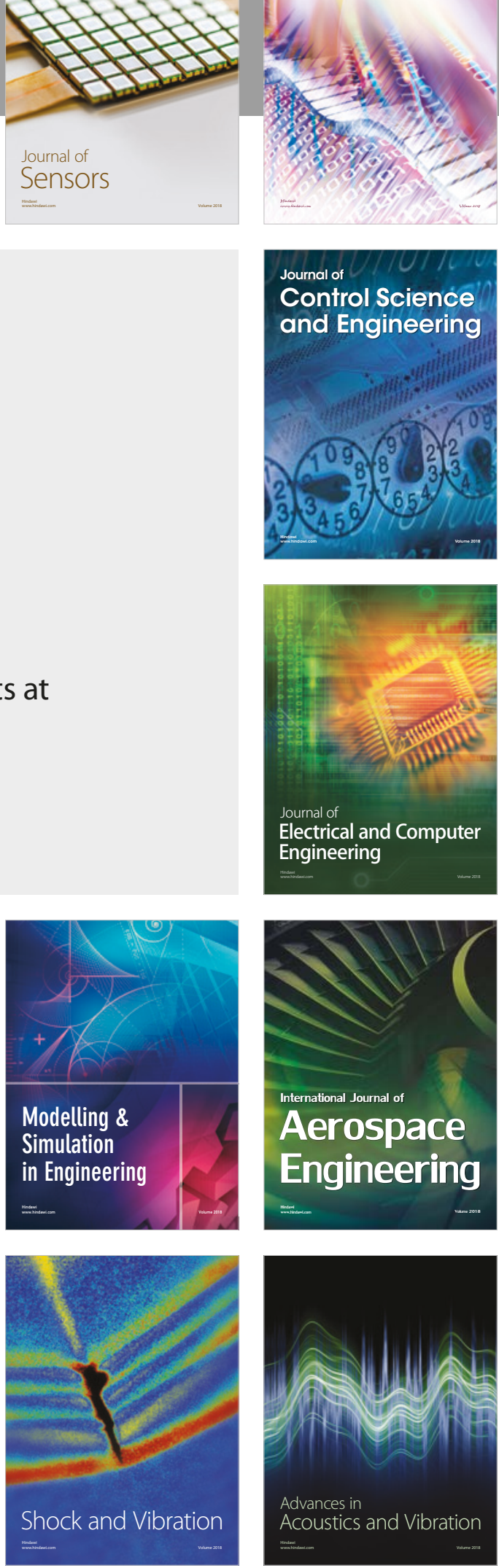